\title{
Variation in Reported Human Head Tissue Electrical Conductivity Values
}

\author{
Hannah McCann ${ }^{1,2}$ (D) Giampaolo Pisano ${ }^{1} \cdot$ Leandro Beltrachini $^{1,2}$
}

Received: 13 December 2018 / Accepted: 13 April 2019 / Published online: 3 May 2019

(c) The Author(s) 2019

\begin{abstract}
Electromagnetic source characterisation requires accurate volume conductor models representing head geometry and the electrical conductivity field. Head tissue conductivity is often assumed from previous literature, however, despite extensive research, measurements are inconsistent. A meta-analysis of reported human head electrical conductivity values was therefore conducted to determine significant variation and subsequent influential factors. Of 3121 identified publications spanning three databases, 56 papers were included in data extraction. Conductivity values were categorised according to tissue type, and recorded alongside methodology, measurement condition, current frequency, tissue temperature, participant pathology and age. We found variation in electrical conductivity of the whole-skull, the spongiform layer of the skull, isotropic, perpendicularly- and parallelly-oriented white matter (WM) and the brain-to-skull-conductivity ratio (BSCR) could be significantly attributed to a combination of differences in methodology and demographics. This large variation should be acknowledged, and care should be taken when creating volume conductor models, ideally constructing them on an individual basis, rather than assuming them from the literature. When personalised models are unavailable, it is suggested weighted average means from the current meta-analysis are used. Assigning conductivity as: $0.41 \mathrm{~S} / \mathrm{m}$ for the scalp, $0.02 \mathrm{~S} / \mathrm{m}$ for the whole skull, or when better modelled as a three-layer skull $0.048 \mathrm{~S} / \mathrm{m}$ for the spongiform layer, $0.007 \mathrm{~S} / \mathrm{m}$ for the inner compact and $0.005 \mathrm{~S} / \mathrm{m}$ for the outer compact, as well as $1.71 \mathrm{~S} / \mathrm{m}$ for the CSF, $0.47 \mathrm{~S} / \mathrm{m}$ for the grey matter, $0.22 \mathrm{~S} / \mathrm{m}$ for WM and 50.4 for the BSCR.
\end{abstract}

Keywords Head conductivity - Electrical impedance tomography $\cdot$ Magnetic resonance electrical impedance tomography . Electroencephalography $\cdot$ Magnetoencephalography $\cdot$ Electromagnetic source localisation

\section{Introduction}

Understanding electrical activity propagation throughout the head is essential in neurophysiology. In particular, forward and inverse solutions for source reconstruction in

Communicated by Jens Haueisen.

Hannah McCann

McCannHM@cardiff.ac.uk

Giampaolo Pisano

PisanoG@cardiff.ac.uk

Leandro Beltrachini

BeltrachiniL@ cardiff.ac.uk

1 School of Physics and Astronomy, Cardiff University, Cardiff, UK

2 Cardiff University Brain Research Imaging Centre (CUBRIC), Cardiff, UK electroencephalography [EEG (Beltrachini, 2019a, b)], magnetoencephalography [MEG (Haueisen et al. 1997; Vorwerk et al. 2014)], transcranial magnetic stimulation [TMS; (Opitz et al. 2011; Salinas et al. 2009] and deep brain stimulation [DBS; (Butson et al. 2007; Dabek et al. 2016; McIntyre et al. 2004)] are governed by such phenomenon. Accurate values of head tissue electrical conductivity are vital to model and localise primary current generators within the brain based on both invasive and non-invasive recordings. Misspecification of tissue conductivities can consequently contribute to significant errors in magnetic field strength and electric surface potential estimations (Cohen and Cuffin 1983a; Haueisen et al. 1995; Okada et al. 1999), which may additionally introduce systemic errors in the EEG and MEG forward problems (Goncalves et al. 2003a, b, c) and result in inaccurate source localisation (Akhtari et al. 2002; Haueisen et al. 2002; Pohlmeier et al. 1997; Vatta et al. 2002). Anwander and colleagues 2002, for example, revealed mean 
EEG source localisation errors of $5.1 \mathrm{~mm}$ and $8.88 \mathrm{~mm}$ for radially- and tangentially-oriented sources, respectively, if white matter (WM) anisotropy was neglected in conductivity models. Whilst Hallez et al. (2005) reported average errors of $11.21 \mathrm{~mm}$, increasing to $13.73 \mathrm{~mm}$ if skull anisotropy in addition to WM anisotropy was not considered. Even accounting for skull anisotropy has yielded maximum localisation errors of $6 \mathrm{~mm}$ (Dannhauer et al. 2011). Miscalculations can further lead to incorrect and inappropriate conclusions made regarding brain function, pathology and disease treatments inferred from E/MEG data (Wendel et al. 2006). Most notably regarding implications in epilepsy treatment (Akhtari et al. 2006; Fabrizi et al. 2006), brain stimulation (De Lucia et al. 2007; Sadleir et al. 2010; Suh et al. 2012) and insights into psychiatric and neurological disorders (Frantseva et al. 2014; Park et al. 2002; Schlosser et al. 2007).

Currently, head tissue conductivity values are often assumed from the literature to create a volume conductor model. Despite extensive research and subsequent review papers (Faes et al.1999; Gabriel et al. 1996a; Geddes and Baker 1967), considerable differences in conductivity are evident between and within reports. Head tissue segmentation is known to be of substantial importance when assigning conductivity values (Akhtari et al. 2000), however there remains discrepancies between such segmentation, for example, consideration of the various layers of the skull (Akhtari et al. 2002), the importance of the dura layer (Ramon et al. 2014) and the influence of blood vessels on high resolution EEG head modelling (Fiederer et al. 2016). Additionally, accounts are inconsistent for the influence of anisotropy on conductivity values (Güllmar et al. 2010; Nicholson, 1965) and the brain-to-skull conductivity ratio [BSCR; (Gutierrez et al. 2004; Wolters et al. 2006)]. Furthermore, existing reports of conductivity vary depending on participant demographics, such as age and pathology, as well as measurement condition (i.e. in vivo, ex vivo or in vitro), applied frequency, tissue temperature and employed methodology.

Unsurprisingly, utilising different methodologies, such as directly applied current (DAC), electrical impedance tomography (EIT), E/MEG, Magnetic Resonance EIT (MREIT) and Diffusion Tensor Imaging (DTI), yield diverse conductivity values. The relative strengths and limitations of these methods is essential to accurately characterise discrepancies and inform future research. With DAC we refer to any invasive method where current is directly applied to tissue, either via multiple implanted electrodes or onto excised tissue, and electrical conductivity is determined from the resulting potential difference between a pair of electrodes. DAC methods have the advantage of not requiring a computational head model, which often introduces simplified assumptions regarding the neurobiology and dynamics of the human head, as well as being cost effective with a low acquisition time, easily portable and useable and has the potential to analyse conductivity of all tissue types. DAC methods however, are invasive, requiring post-mortem samples or excised tissues that are not under biophysically natural conditions. Tissues obtained post-mortem, for example, are subject to biochemical processes initiated by death, such as changes in ion mobility and cell membrane polarisation, which consequently affect conductivity (Opitz et al. 2017). Opitz and colleagues (2017) importantly demonstrated, despite controlling for confounding variables (i.e. temperature), that live and post-mortem intracranial electrical fields significantly differed. Similarly, excised tissues undergo various extracting, preservation and holding procedures (i.e. saline soaked, time since excision, etc.) which can change the electrolyte concentration (Akhtari et al. 2002) and hence influence conductivity. On the other hand, EIT, E/MEG, MREIT and DTI methods are non-invasive and occur in vivo, having the advantage of remaining under natural conditions. Additionally, EIT and E/MEG are both portable and cost effective with low acquisition times, compared to MREIT and DTI methods which are non- portable, more expensive and with high acquisition times, but EIT and E/MEG have lower spatial resolution than MREIT and DTI and require the use of a computational head model. Both MREIT and DTI however, employ magnetic resonance (MR) imaging, making skull conductivity non-accessible due to weak MR signal towards bone layers. A further advantage of DTI is the ability to classify anisotropic and heterogenous conductivity values of soft tissues (Johansen-Berg and Behrens 2013). A summary of the strengths and weaknesses of the described methods are provided in Table 1.

Considering the vast disparity in data, assuming conductivity from previous literature is insufficient when accurate and precise values are required. Significant and important factors affecting this variation, however, are currently unknown. Knowledge of influential variables, such as tissue segmentation, methodology employed, sample temperature or participant pathology can provide insights into the stability of tissue conductivity values and methodology, as well as suggest areas for future research. The current study aimed to systematically and extensively investigate all published reports of human head tissue electrical conductivity to (i) evidence any significant variations in conductivity values of different head tissue types; (ii) determine any significant factors contributing to variation; and (iii) analyse the impact these factors may have on source reconstruction in E/MEG. A systematic review, restricted to human head tissue, was carried out to identify relevant papers, and a meta-analysis was completed to reveal significant factor variables via a multiple regression. It was hypothesised that head tissue conductivity would vary between and within tissues. It was expected the meta-analysis would further reveal significant influential factors and their impact. 
Table 1 Methodology strengths and limitations

\begin{tabular}{lll}
\hline Method & Strengths & Limitations \\
\hline DAC & - No computational head & - Invasive \\
& model required & - Unnatural conditions if not \\
& - Potential to classify all & in vivo \\
& tissue types & - Homogeneous \\
& - Portable & \\
& - Cost effective & \\
& - Low acquisition time & \\
EIT & - Non-invasive & - Computational head model \\
& - In vivo & required \\
& - Portable & - Low spatial resolution \\
& - Cost effective & - Low signal-to-noise \\
& - Low acquisition time & - Homogeneous \\
E/MEG & - non-invasive & - Computational head model \\
& - in vivo & required \\
& - portable & - Low spatial resolution \\
& - cost effective & - homogeneous \\
& - low acquisition time & \\
MREIT & - Non-invasive & - Low signal-to-noise ratio \\
& - In vivo & - Weak MR signal in skull layers \\
& - High spatial resolution & - Non-portable \\
& - Anisotropic & - Relatively expensive \\
& & - High acquisition time \\
DTI & - Non-invasive & - Non-portable \\
& - In vivo & - Relatively expensive \\
& - High spatial resolution & - Weak MR signal in skull layers \\
& - Anisotropic & - High acquisition time \\
& - Heterogeneous & \\
\hline & & \\
\hline
\end{tabular}

\section{Methods}

\section{Literature Search}

Preferred Reporting Items for Systematic reviews and MetaAnalyses (PRISMA) statement guidelines (Moher et al. 2009) were followed and a PRISMA checklist and flow diagram were completed (Appendix 1) An extensive literature search, spanning three databases (PubMed, Scopus and Web of Knowledge), was conducted to retrieve published and peer-reviewed studies exploring electrical conductivity (or equivalent) of the human head (or equivalent). The keywords utilised for the systematic literature search are provided in Appendix 2. Article titles were systematically searched using relevant and/or equivalent keywords, unrestricted by year of publication, language or design. Reference lists of included papers were hand-searched to identify additional papers. Duplicates following the initial literature search were removed.

\section{Selection Criteria}

Papers met the inclusion criteria if they (i) provided at least one conductivity measure (or equivalent from which conductivity could be calculated), of the (ii) human (iii) head, where (iv) employed methodology and (v) tissue type were available. Reviews were only included as an information source to the original reference, where data was thus extracted. Exclusions were made if any of the five inclusion criteria were absent or ambiguous, or if an English version was unavailable after extensive search. In addition to conductivity value, methodology and tissue type, reports were collected on measurement condition (i.e. in vivo, ex vivo, or in vitro), applied frequency to determine the conductivity, tissue temperature, as well as participant's age, gender and pathology. Missing information for one or more of these variables did not result in exclusion. Studies applying frequencies above $1 \mathrm{kHz}$ were excluded from analysis on the grounds this frequency is besides the scope of typical brain activity recorded in E/MEG.

All identified titles resulting from the literature search, following removal of duplicates, were initially screened for applicability and/or immediate exclusion. Remaining abstracts were further assessed, and full texts of potentially relevant papers were obtained to determine if they consequently met the inclusion criteria.

\section{Data Extraction and Synthesis}

All conductivity, resistivity or impedance values were extracted from each paper and converted to $\mathrm{S} / \mathrm{m}$ for standardisation. The sample mean and standard deviation were subsequently calculated for every differentiation in methodology within each paper and characterised according to the aforementioned variables.

\section{Variable Definitions and Classification}

\section{Tissue Types}

For the current review, tissues were separated into four major compartments, each comprised of sub-compartments: the scalp (skin, fat, muscle), the skull (spongiform, inner and outer compact bone and sutures), cerebrospinal fluid $(C S F)$ and the brain [grey matter $(G M), W M$, the dura layer, blood, cerebellum, lesions, epileptogenic zone (EZ)]. Conductivity values were assigned according to tissue type as reported. Tissues were classified as whole-scalp, whole-skull or whole-brain when no conductivity values for their subcompartments were reported, similarly whole-compact bone was assigned if no values for the inner and outer compact bone were provided. If given, WM was further segmented into WM oriented in parallel (WM_par) or perpendicular (WM_perp) to the applied current. See Fig. 1 for a detailed representation of all tissue compartments. Additionally, when available, the $B S C R$ was reported as a nominal ratio without units. 
Fig. 1 Figure displaying the various tissue compartments of the head and a subfigure of the detailed layers of the scalp, skull and brain
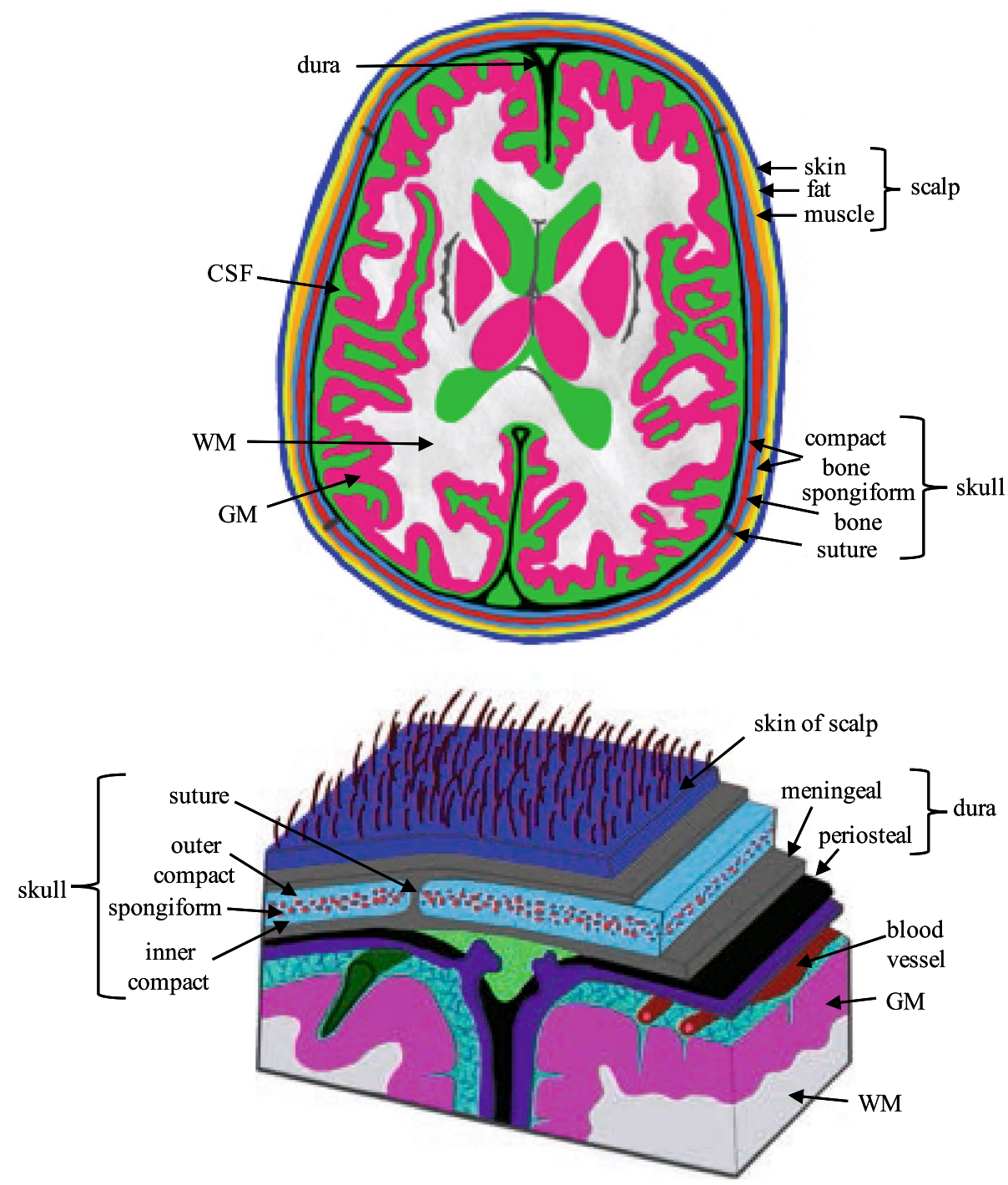

\section{Measurement Conditions}

Conditions were separated into three main categories:

In vivo- "within the living"; experiment conducted on or in whole living organisms/cells. Electrical conductivity values obtained within a living head were considered in vivo.

Ex vivo-_out of the living"; experiment in or on tissue from an organism in an external environment, but with minimal alteration of natural conditions, e.g. cultured cells derived from biopsies. Experiments where tissue was excised but kept within conditions similar to the human head were characterised as ex vivo.

In vitro-"within the glass"; experiment within a controlled artificial environment outside of a living organism, isolated from their usual biological surroundings e.g. in a test tube/dish. Measurements where tissue was excised and stored in environments unlike the human head were classified as in vitro.

\section{Measurement Methods}

Data acquisition techniques were categorised into five groups:

$D A C$-invasive method of determining electrical conductivity, where a current was directly applied to the tissue, either via implanted electrodes in the head, or onto excised samples. The resulting electric potential difference from the applied current is measured via additional (implanted or applied to excised tissue) electrodes to calculate the electrical conductivity. Studies where electrical current was directly and invasively applied to the head tissue were characterised as DAC.

EIT - a non-invasive medical imaging technique where alternating current at single or multiple frequencies is applied to the skin through two or more conducting surface electrodes. The resulting potential difference between the remaining measuring electrodes is then recorded. From this the electrical conductivity, permittivity and impedance 
can be inferred to create a tomographic image (Barber and Brown 1984; Henderson and Webster 1978). Papers indicating an applied current of less than $1 \mathrm{kHz}$, injected through any number of electrodes and the resulting voltage were classified as EIT.

MREIT - measures the induced magnetic flux density resulting from an injected current (as in EIT) using a magnetic resonance imaging (MRI) scanner. The internal current density is then computed and combined with magnetic flux density measurements to perform conductivity map reconstruction, using various inverse solutions (Bodenstein et al. 2009). Studies specifying acquisition of MRI data during current injection (as in any EIT method) to reconstruct conductivity (using any inverse method) were categorised as MREIT.

$E / M E G$ - electromagnetic data recorded from E/MEG employed to iteratively estimate the equivalent electrical conductivity that best matches the computed source localisation given the obtained E/MEG data Baysal and Haueisen (2004a, b). Articles estimating conductivity by employing data from E/MEG (of any set up) were characterised as E/ MEG.

DTI-diffusion-weighted MR images of the brain are acquired to measure the diffusion tensor eigenvalues, from which the electrical conductivity tensor eigenvalues are directly calculated (Sekino et al. 2005; Tuch et al. 1999; Tuch et al. 2001a, b). Texts using diffusion imaging (of any protocol) to explicitly estimate the electrical conductivity tensor map were considered as employing DTI methodology for the current review. This included any method for estimating conductivity from the diffusion tensor. DTI papers where conductivity was not explicitly reported were not included in the current review.

\section{Frequency}

Frequency of applied or injected current (if applicable). Frequency was not extracted from papers where this was not specified.

\section{Temperature}

Classified according to whether the tissue sample was measured at/near body temperature $\left(37^{\circ} \mathrm{C}\right)$ or room temperature $\left(18-25^{\circ} \mathrm{C}\right)$. Unknown values were not reported for analysis.

\section{Participant's Age}

When available, mean and standard deviation of participant's age were calculated for each paper and recorded in Table 2 . Age at time of death was recorded for deceased participants. If specific age was unavailable, age was characterised as adult (all participants were over the age of 18), paediatric (all participants were under the age of 18), or both (participants were a mixture of over and under the age of 18).

\section{(g) Participant Pathology}

Participants were characterised as healthy if they had no neurological, developmental or psychological deficits, as reported in the research paper. Pathology was categorised as epilepsy for studies recruiting patients that presented with any classification of epileptic seizure. Similarly, tumour was assigned to papers where patients displayed any type of tumour, and neuro to patients with any type of neurological disorder that was not otherwise classifiable. Further pathologies included Parkinson's Disease, Alzheimer's Disease and stroke. All conductivity values were assumed to originate from healthy tissue, within the classified pathology, unless otherwise stated. Pathology was reported as unknown if not available in the literature.

\section{Quality Analysis}

Drawing robust conclusions from systematic reviews and meta-analyses requires consideration of the systematic and random errors introduced in each included study by "assessing the methodological quality" (Moher et al. 1996; Verhagen et al. 2001) in order to estimate "risk of bias". Various tools are available for assessing study quality and addressing the systematic errors in each study, however none specifically to assess the quality of studies measuring the electrical conductivity of the human head. The current meta-analysis therefore, made use of the Cochrane Collaboration recommended Quality Assessment of Diagnostic Accuracy Studies (QUADAS) checklist (Whiting et al. 2003), where each item was adjusted for relevance, and any additional relevant items were added. A scaled numerical value was further assigned according to the studies compliance with each item; any irrelevant items were ignored. The sum, divided by the number of relevant items, was subsequently calculated to provide a final Quality Assessment Score (QAS), with an absolute maximum value of one (the closer the score is to one, the more reliable the study was considered). To ensure reliability of the QAS's, papers were chosen at random and QASs calculated by two researchers, any discrepancies were discussed and if not resolved the mean QAS was assigned. The employed Quality Assessment Protocol and three examples are provided in Appendix 3.

In addition to accounting for systematic errors within each study, random errors produced from inherently unpredictable variation in methodology were accounted for. This was adapted from the guidelines provided by Rosenthal (1991) and Borenstein and colleagues (2011) for metaanalysis weighting. Confidence values for each measurement were calculated to indicate the confidence each value 
Table 2 Summary of papers included in meta-analysis

\begin{tabular}{|c|c|c|c|c|c|c|c|}
\hline Author & Method & Design & Freq. (Hz) & Participants & Age (years) & Pathology & Weight \\
\hline $\begin{array}{l}\text { (Burger and van Milaan } \\
\text { 1943) }\end{array}$ & DAC & Ex vivo & 0 & $\mathrm{n}=1$ & Adult & Healthy & 0.799 \\
\hline (Rosenthal and Tobias 1948) & DAC & Ex vivo & 1000 & $\mathrm{n}=1$ & Adult & Healthy & 0.361 \\
\hline $\begin{array}{l}\text { (Burger and Van Dongen } \\
\text { 1961) }\end{array}$ & DAC & Ex vivo & 1000 & $\mathrm{n}=1$ & Adult & Healthy & 0.444 \\
\hline (Rush and Driscoll 1968) & DAC & Ex vivo & & $\mathrm{n}=1$ & Adult & Healthy & 0.833 \\
\hline (Cohen and Cuffin 1983b) & E/MEG & In vivo & $0.3-300$ & $\mathrm{n}=2(\mathrm{~m})$ & Adult & Healthy & 0.705 \\
\hline (Eriksen 1990) & E/MEG & In vivo & 40 & $\mathrm{n}=4$ & Adult & Healthy & 0.221 \\
\hline (Haacke et al. 1991) & MREIT & In vivo & & $\mathrm{n}=3$ & Adult & Healthy & $0.852 \pm 0.02$ \\
\hline (Law 1993) & DAC & In vitro & 100 & $\mathrm{n}=1$ & Adult & Healthy & 0.8723 \\
\hline (Pierpaoli et al. 1996) & DTI & In vivo & & $\mathrm{n}=8$ & Adult & Healthy & 0.344 \\
\hline (Baumann et al. 1997) & DAC & In vitro & $10-10 \mathrm{kHz}$ & $\mathrm{n}=7(3 \mathrm{~m})$ & 6.6 & Neuro & $0.69 \pm 0.051$ \\
\hline (Sorensen et al. 1999) & DTI & In vivo & & $\mathrm{n}=1$ & Adult & Stroke & 0.814 \\
\hline (Uluğ and Van Zijl 1999) & DTI & In vivo & & $\mathrm{n}=5$ & Adult & Healthy & 0.375 \\
\hline (Oostendorp et al. 2000) & DAC & In vitro & $10-100$ & $\mathrm{n}=1, \mathrm{n}=2(1 \mathrm{~m})$ & Adult & Healthy & 0.768 \\
\hline (Akhtari et al. 2000) & DAC & In vitro & 20 & $\mathrm{n}=1$ & Adult & Healthy & 0.855 \\
\hline (Akhtari et al. 2002) & DAC & Ex vivo & 10,90 & $\mathrm{n}=4(2 \mathrm{~m})$ & $56 \pm 26.7$ & Epilepsy & 0.931 \\
\hline (Hoekema et al. 2003) & DAC & In vitro, ex vivo & 10 & $n=1(f), n=5$ & $68,33.6 \pm 15.9$ & Healthy & 0.855 \\
\hline (Goncalves et al. 2003b) & EIT & In vivo & 60 & $\mathrm{n}=6(3 \mathrm{~m})$ & $32.3 \pm 7$ & Healthy & 0.62 \\
\hline (Goncalves et al. 2003a) & EIT \& E/MEG & In vivo & 60 & $\mathrm{n}=6(3 \mathrm{~m})$ & Adult & Healthy & $0.496 \pm 0.006$ \\
\hline $\begin{array}{l}\text { (Baysal and Haueisen 2004a, } \\
\text { b) }\end{array}$ & E/MEG & In vivo & 4 & $\mathrm{n}=10(5 \mathrm{~m})$ & $30 \pm 13$ & Healthy & $0.365 \pm 0.368$ \\
\hline (Gutierrez et al. 2004) & E/MEG & In vivo & 2 & $\mathrm{n}=2(1 \mathrm{~m})$ & $32.5 \pm 10.6$ & Healthy & $0.52 \pm 0.08$ \\
\hline (Clerc et al. 2005) & EIT & In vivo & 110 & $\mathrm{n}=1$ & Adult & Healthy & $0.639 \pm 0.009$ \\
\hline (Sekino et al. 2005) & DTI & In vivo & & $\mathrm{n}=5$ & Adult & Healthy & $0.672 \pm 0.02$ \\
\hline (Lai et al. 2005) & EIT & In vivo & 50 & $\mathrm{n}=5(4 \mathrm{~m})$ & $10 \pm 2$ & Epilepsy & 0.544 \\
\hline (Zhang et al. 2006) & EIT & In vivo & 50 & $\mathrm{n}=2$ & Paediatric & Epilepsy & 0.656 \\
\hline (Akhtari et al. 2006) & DAC & Ex vivo & $5-1005$ & $\mathrm{n}=21(12 \mathrm{~m})$ & $13.5 \pm 15.1$ & Epilepsy & 0.946 \\
\hline (Tang et al. 2008) & DAC & In vitro & $1 \mathrm{kHz}$ & $\mathrm{n}=48(38 \mathrm{~m})$ & 47.6 & Healthy & 0.999 \\
\hline (Gattellaro et al. 2009) & DTI & In vivo & & $\mathrm{n}=20(10 \mathrm{~m})$ & $60.95 \pm 11.9$ & Healthy, PD & 0.344 \\
\hline (Rullmann et al. 2009) & DTI & In vivo & & $\mathrm{n}=1$ & 0.916 & Epilepsy & 0.975 \\
\hline (Voigt et al. 2009) & MREIT & In vivo & & $\mathrm{n}=1$ & Adult & Healthy & $0.578 \pm 0.067$ \\
\hline (Akhtari et al. 2010) & DAC & Ex vivo & $6-1005$ & $\mathrm{n}=15(8 \mathrm{~m})$ & $7.93 \pm 6.04$ & Epilepsy & 0.946 \\
\hline (Van Lier et al. 2011) & MREIT & In vivo & & $\mathrm{n}=1$ & 46 & Tumour & $0.559 \pm 0.014$ \\
\hline (Güllmar et al. 2010) & DTI & In vivo & & $\mathrm{n}=1(\mathrm{~m})$ & 30 & Healthy & 0.406 \\
\hline (Wang et al. 2010) & DTI & In vivo & & $\mathrm{n}=71(39 \mathrm{~m})$ & $41.8 \pm 14.5$ & Healthy & 0.375 \\
\hline (Dannhauer et al. 2011) & E/MEG & In vivo & & $\mathrm{n}=4$ & $25 \pm 4.6$ & Healthy & 0.34 \\
\hline (Voigt et al. 2011) & MREIT & In vivo & & $\mathrm{n}=6(\mathrm{~m})$ & $37 \pm 6$ & Healthy & $0.846 \pm 0.042$ \\
\hline (van Lier et al. 2012) & MREIT & in vivo & & $\mathrm{n}=1$ & 65 & Stroke & 0.475 \\
\hline (Huhndorf et al. 2013) & MREIT & In vivo & & $\mathrm{n}=12$ & & Tumour & $0.517 \pm 0.259$ \\
\hline (Zhang et al. 2013) & MREIT & In vivo & & $\mathrm{n}=3$ & Adult & Healthy & 0.731 \\
\hline (Aydin et al. 2014) & E/MEG & In vivo & & $n=1(f)$ & 17 & Epilepsy & 0.86 \\
\hline (Kim et al. 2014) & MREIT & In vivo & & $\mathrm{n}=1$ & Adult & Healthy & $0.805 \pm 0.117$ \\
\hline $\begin{array}{l}\text { (Ouypornkochagorn et al. } \\
\text { 2014) }\end{array}$ & EIT & In vivo & & $\mathrm{n}=1$ & Adult & Healthy & $0.774 \pm 0.01$ \\
\hline (Lee et al. 2015) & MREIT & In vivo & & $\mathrm{n}=2$ & Adult & Healthy & 0.787 \\
\hline (Ropella and Noll, 2017) & MREIT & In vivo & & $\mathrm{n}=4$ & Adult & Healthy & $0.028 \pm 0.052$ \\
\hline (Dabek et al. 2016) & EIT & In vivo & 2 & $\mathrm{n}=9(4 \mathrm{~m})$ & $32.5 \pm 10$ & Healthy & $0.627 \pm 0.037$ \\
\hline (Akhtari et al. 2016) & DAC & In vitro & 10 & $\mathrm{n}=24$ & Paediatric & Epilepsy & $0.698 \pm 0.212$ \\
\hline (Acar et al. 2016) & E/MEG & In vivo & & $\mathrm{n}=2(\mathrm{~m})$ & $21.5 \pm 2.12$ & Healthy & $0.718 \pm 0.019$ \\
\hline
\end{tabular}


Table 2 (continued)

\begin{tabular}{|c|c|c|c|c|c|c|c|}
\hline Author & Method & Design & Freq. (Hz) & Participants & Age (years) & Pathology & Weight \\
\hline (Gurler and Ider 2017) & MREIT & In vivo & & $\mathrm{n}=1$ & 23 & Healthy & $0.817 \pm 0.218$ \\
\hline (Lee et al. 2016) & MREIT & In vivo & & $\mathrm{n}=1$ & Adult & Healthy & $0.561 \pm 0.382$ \\
\hline (Koessler et al. 2017) & EIT & In vivo & 50 & $\mathrm{n}=15(10 \mathrm{~m})$ & $38 \pm 10$ & Epilepsy & $0.643 \pm 0.0478$ \\
\hline (Huang et al. 2017) & EIT & In vivo & $1-100$ & $\mathrm{n}=10$ & Adult & Epilepsy & 0.613 \\
\hline $\begin{array}{l}\text { (Fernández-Corazza et al. } \\
\text { 2017) }\end{array}$ & EIT & In vivo & 27 & $\mathrm{n}=4(\mathrm{~m})$ & $49 \pm 4.8$ & Healthy & $0.593 \pm 0.078$ \\
\hline (Hampe et al. 2018) & MREIT & In vivo & & $\mathrm{n}=4$ & $39.5 \pm 3.4$ & Healthy & 0.406 \\
\hline (Arumugam et al. 2017) & EIT & In vivo & 27 & $\mathrm{n}=10$ & & Healthy & 0.292 \\
\hline (Michel et al. 2017) & MREIT & In vivo & & $\mathrm{n}=1$ & 29 & Healthy & $0.89 \pm 0.028$ \\
\hline (Tha et al. 2018) & MREIT & In vivo & & $\mathrm{n}=30(14 \mathrm{~m})$ & $50.7 \pm 18.2$ & Tumour & 0.486 \\
\hline (Chauhan et al. 2018) & DTI & In vivo & 10 & $\mathrm{n}=2(\mathrm{~m})$ & & Healthy & 0.939 \\
\hline
\end{tabular}

Method: $D C$ direct current, EIT electrical impedance tomography, E/MEG electro- or magneto-encephalography, MREIT magnetic resonance EIT, DTI diffusion tensor imaging. Frequency (Hz, unless stated otherwise. Participants: number $(\mathrm{n}=)$, male/female $(\mathrm{m} / \mathrm{f})$. Age: mean \pm standard deviation, unless stated otherwise. Pathology: neurological disorder (neuro), Parkinson's Disease (PD). Weight: mean \pm standard deviation

of conductivity was $100 \%$ accurate. Firstly, the relative error was calculated for each conductivity value, as the standard deviation percentage of a multitude of values for a single tissue type for each participant (if the method is $100 \%$ precise, each value for the same tissue should be the same) or the error attributed to the measurement protocol-both described as a decimal. If both the standard deviation and measurement error were provided, the standard deviation was used to calculate the relative error. The relative error was then subtracted from one (where one indicates complete confidence the conductivity value is $100 \%$ accurate) to obtain a final confidence value of which the maximum is one. For example, a reported conductivity value with an associated standard deviation percentage of $8 \%$ will receive a confidence value of 0.92 . Alternatively, when the standard deviation was not provided, the experimental error was utilised instead; e.g. a study with a methodological error of 0.05 would receive a confidence value of 0.95 .

To incorporate both the systematic and random errors associated with each study, the Quality Assessment Score of each study and the confidence values of each conductivity value were combined to provide a "weight". This weight was calculated by multiplying the QAS by the confidence value (both with a maximum of one). The maximum associated weight each value has towards the analysis is therefore one. Values assigned weights closer to one were therefore regarded as being more accurate.

\section{Statistical Analysis}

Data was pooled and grouped according to tissue type, in order to determine (i) the variation in conductivity for each tissue, (ii) which significant variables account for differences in conductivity, (iii) whether mean conductivity values for each tissue type are statistically different depending on employed methodology and participant demographics, and (iv) reveal any statistical relationship between conductivity and reported variables.

Boxplot diagrams, presenting the range, median and mean of conductivity measurements for each tissue type were created to demonstrate variation in conductivity within different tissues. For each tissue with more than three results in at least two variables, a weighted multiple regression was carried out using SPSS (Corp 2013). The dependent variable (conductivity) was regressed against every independent variable (IV; measurement condition, method, frequency, temperature, age and pathology) collectively, to determine the proportion of variance accounted for by all factors, and individually to discover significant factors predicting variation in conductivity. Weights for each conductivity value were assigned according to the Quality Analysis described above (Sect. 2.5). A two-tailed $t$ test (when comparing two independent variables) or a one-way Analysis of Variance (ANOVA; when comparing more than two independent variables) was conducted to reveal differences in conductivity for each tissue, according to categorical IV's previously revealed to account for a significant proportion of variance. A Pearson correlation analysis was alternatively conducted for continuous IV's accounting for significant variation to reveal any statistical relationships.

\section{Results}

\section{Search Results}

Following removal of duplicates, 3121 studies were identified through the literature and reference list search, of which 382 abstracts were screened for relevance and 211 full text articles were obtained and assessed for eligibility. A total 
Table 3 Descriptive statistics for each tissue type

\begin{tabular}{|c|c|c|c|c|c|c|c|c|}
\hline & Minimum & Maximum & Mean & Weighted mean & Standard deviation & n. values & n. studies & n. participants \\
\hline Scalp & 0.137 & 2.1 & 0.5345 & 0.4137 & 0.1760 & 44 & 10 & 44 \\
\hline Fat & & & 0.6 & & & 1 & 1 & 1 \\
\hline Muscle & 0.1482 & 0.4167 & 0.3243 & 0.3243 & 0.1526 & 3 & 1 & 1 \\
\hline Whole skull & 0.0182 & 1.718 & 0.0708 & 0.0160 & 0.019 & 99 & 20 & 121 \\
\hline Spongy & 0.0012 & 0.2890 & 0.0559 & 0.0497 & 0.0735 & 18 & 5 & 58 \\
\hline Compact & 0.0024 & 0.0079 & 0.0045 & 0.0046 & 0.0016 & 9 & 4 & 54 \\
\hline Outer compact & 0.0008 & 0.0078 & 0.0047 & 0.0049 & 0.0029 & 10 & 2 & 5 \\
\hline Inner compact & 0.0028 & 0.0129 & 0.0067 & 0.0068 & 0.0036 & 10 & 2 & 5 \\
\hline Sutures & 0.0078 & 0.0735 & 0.0273 & 0.0266 & 0.0239 & 6 & 2 & 49 \\
\hline CSF & 1 & 2.51 & 1.6922 & 1.71 & 0.2981 & 43 & 14 & 37 \\
\hline Whole brain & 0.054 & 13.75 & 1.059 & 0.3746 & 0.1322 & 63 & 11 & 70 \\
\hline GM & 0.06 & 2.47 & 0.5981 & 0.4660 & 0.2392 & 66 & 16 & 153 \\
\hline WM & 0.0646 & 0.81 & 0.24 & 0.2167 & 0.1703 & 104 & 15 & 106 \\
\hline WM_perp & 0.0620 & 0.4390 & 0.1216 & 0.1175 & 0.0495 & 41 & 3 & 49 \\
\hline WM_par & 0.0543 & 0.9150 & 0.1352 & 0.1226 & 0.0929 & 41 & 3 & 49 \\
\hline Blood & 0.433 & 07622 & 0.5799 & 0.5737 & 0.106 & 14 & 3 & 3 \\
\hline EZ & 0.2320 & 0.5278 & 0.2994 & 0.2949 & 0.0737 & 15 & 1 & 15 \\
\hline Dura & & & 0.461 & & & 1 & 1 & 2 \\
\hline Cerebellum & 0.391 & 0.635 & 0.5415 & 0.5370 & 0.1141 & 4 & 1 & 1 \\
\hline Lesions & 0.1 & 1.77 & 0.8087 & 0.8757 & 0.3772 & 19 & 5 & 45 \\
\hline BSCR & 17.9 & 290 & 58.69 & 50.4 & 38.93 & 51 & 10 & 47 \\
\hline
\end{tabular}

Spongiform (Spongy) and Compact (Inner and Outer Compact) of the skull layer, CSF cerebrospinal fluid, GM grey matter, $W M$ white matter, $B S C R$ brain-scalp conductivity ratio, number of... (n.)

of 155 papers were excluded (see Appendix 1, Figure A. 1: PRISMA flow diagram).

\section{Included studies}

A total of 56 studies (407 participants) were included in the quantitative synthesis (Table 2). Seventeen different tissue types were identified, using five methodologies and three measurement conditions. Conductivity was measured in vivo in 42, in vitro in seven and ex vivo in eight research papers. Measurements were obtained using DAC in 14 studies, using EIT in 10, E/MEG in 7, MREIT in 15 and DTI in 9 papers. Conductivity was acquired at frequencies varying between 0 and $1005 \mathrm{~Hz}$, and tissue temperatures between 18.5 and $37.5{ }^{\circ} \mathrm{C}$. Of the 23 articles that specified, total participant age ranged from 4 months to 87 years old, whilst the remainder classified subjects into adults or children. Forty papers reported on healthy participants, participants from ten studies were diagnosed with epilepsy, patients with tumours were included in three studies, stroke patients were employed in two papers, whilst separate papers included patients with various neurological disorders and Parkinson's Disease. Descriptive statistics for each tissue type are provided (Table 3), in addition to a boxplot displaying variation in conductivity values for different tissue types
(Fig. 2). The average mean was calculated for each tissue type, where all conductivity values contributed equally to the mean. A weighted average mean was additionally calculated to take into consideration the quality of each study and provide a recommended value that was obtained under suitable and realistic conditions. The weighted average mean and standard deviation (in $\mathrm{S} / \mathrm{m}$ ) for the main tissue types were: scalp $=0.41 \pm 0.18$, whole skull $=0.02 \pm 0.02$, spongiform skull layer $=0.048 \pm 0.07$, whole compact skull layer $=0.005 \pm 0.002$, outer compact $=0.005 \pm 0.003$, inner compact $=0.007 \pm 0.004, \quad \mathrm{CSF}=1.71 \pm 0.3$, $\mathrm{GM}=0.47 \pm 0.24, \mathrm{WM}=0.22 \pm 0.17, \mathrm{BSCR}=50.4 \pm 39$. A boxplot evidencing the average weights assigned to each study according to the employed methodology is further demonstrated (Fig. 3). Average study weights were revealed to be significantly different depending on methodology [F $(4,56)=3.121, \mathrm{p}=.022)]$.

Following visual inspection, it can be seen conductivity values vary considerably within and between tissue types. Insufficient data was available to calculate regression statistics for muscle, fat, blood, the epileptogenic zone, the dura layer and the cerebellum. 
Variation in conductivity values for all tissues and brain-to-skull conductivity ratio
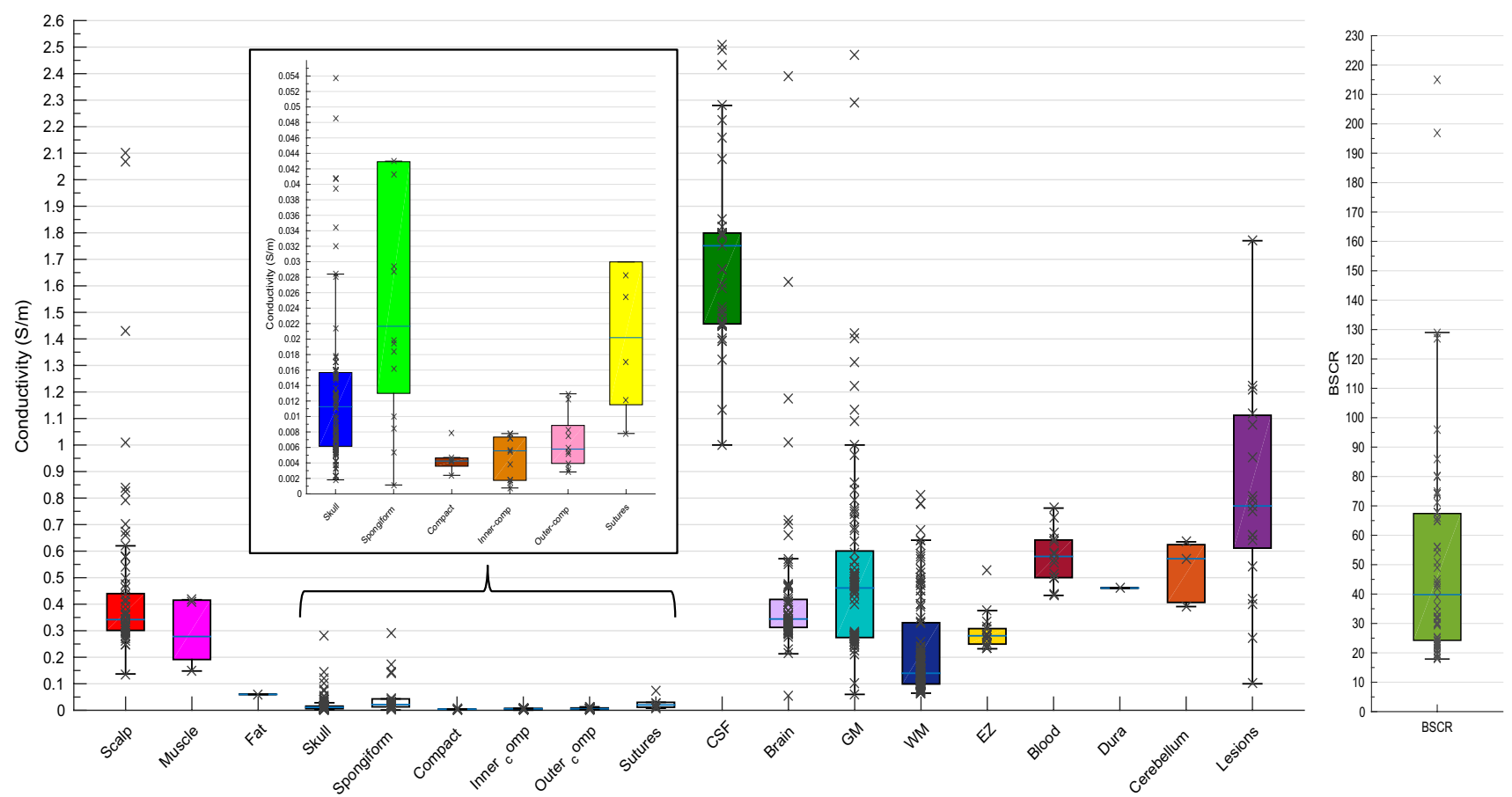

Fig. 2 Boxplot displaying the inter-quartile range (first to third quartile as solid box), the median (solid blue line), minimum and maximum (solid whiskers) of all available conductivity values $(\mathrm{S} / \mathrm{m})$ for all tissues and BSCR displayed as crosses

\section{Scalp}

A weighted multiple regression revealed scalp conductivity variation was insignificantly predicted by the IV's collectively ( $\mathrm{p}>.05)$. Although insignificantly different, a comparison between employed method (as shown in Fig. 4) was made to graphically display any elevated values and further demonstrate variation despite statistical insignificance. Figure 4 further reveals less deviation within values for EIT than for E/MEG. Huang and colleagues (2017) yielded conductivity measurements significantly above the interquartile range. Additionally, Baysal and Haueisen (2004a, b) revealed highly elevated conductivity values beyond the axis range displayed in Fig. 4, with standard deviation $>5000 \%$ (see Sect. 4.1 for further explanation of outliers).

\section{Skull}

\section{Whole-Skull}

A weighted multiple regression revealed deviation in wholeskull conductivity could be significantly predicted by the methodology, condition, temperature, frequency, pathology and age collectively $\left[\mathrm{R}^{2}(6,36)=.827, \mathrm{p}<.001\right]$. A one-way ANOVA revealed conductivity of the whole skull varied significantly according to employed methodology [Fig. 5; F(2,
$96)=4.088, \mathrm{p}=.020]$. Differences in conductivity values for the whole-skull were statistically different according to method, where values obtained using EIT were significantly lower than those obtained with DAC and E/MEG.

\section{Spongiform Bone Skull Layer}

A weighted multiple regression revealed variation in conductivity values of the spongiform bone layer of the skull was significantly predicted by condition, temperature, frequency, pathology and age $\left[\mathrm{R}^{2}(5,6)=.832, \mathrm{p}=.026\right]$. Spongiform conductivity measurements were significantly different according to condition [Fig. 6; $\mathrm{F}(2,15)=11.357$, $\mathrm{p}=.001]$ and temperature $[\mathrm{t}(16)=2.449, \mathrm{p}=.001)$.

\section{Compact Bone Skull Layer}

None of the IV's significantly predicted variation in conductivity values of the whole compact layer, the inner compact bone layer or the outer compact bone layer according to the weighted multiple regression analysis. Despite insignificant results, a graphical representation of conductivity for the different compact bone layers revealed clear diversions within and between each of the layers (Fig. 7). 


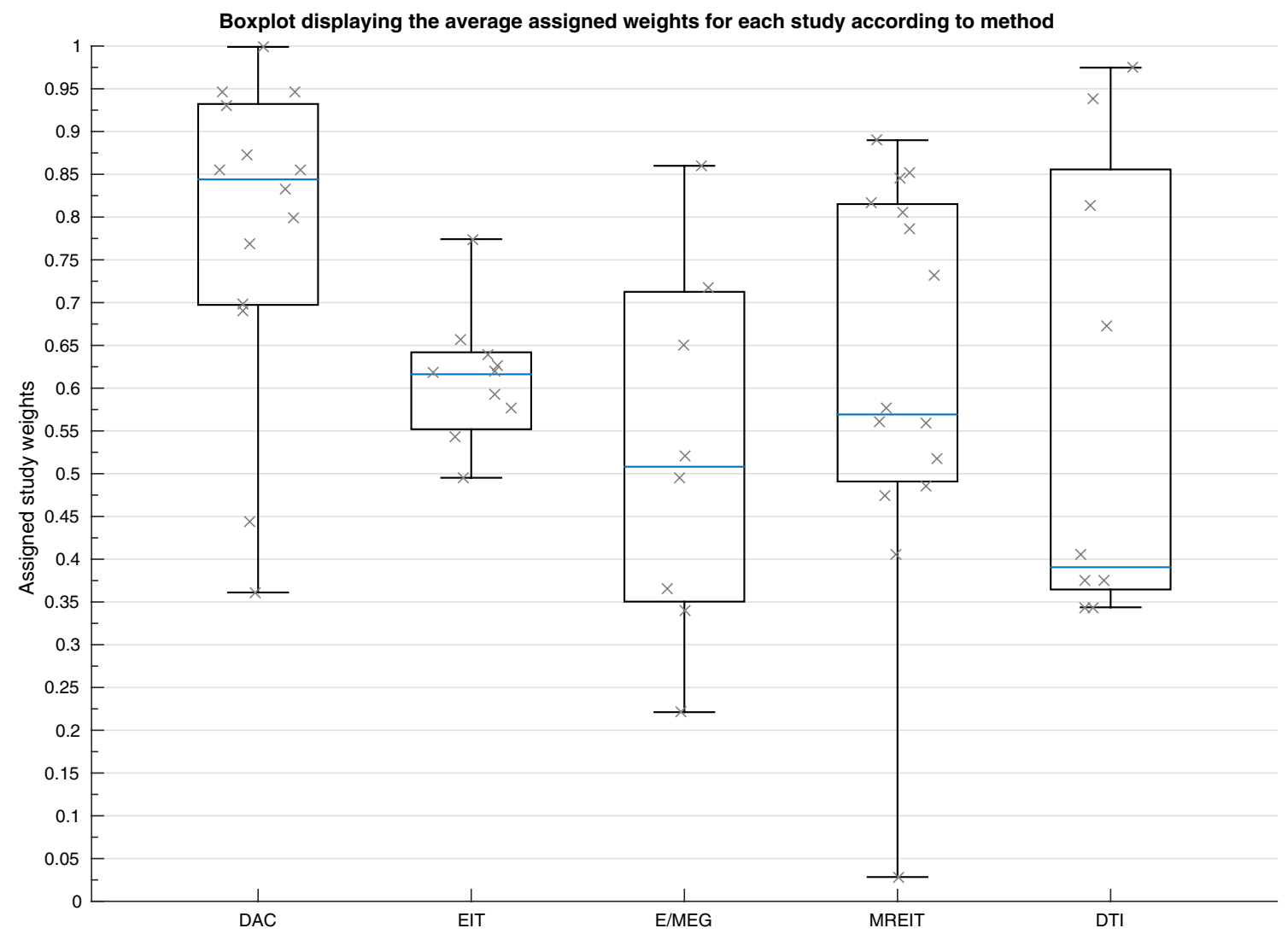

Fig. 3 Boxplot displaying the mean value of the assigned weights for each study (indicated by a cross) dependent on the employed methodology

\section{Cerebrospinal Fluid (CSF)}

Significant differences $[\mathrm{t}(36)=2.695, \mathrm{p}=.006]$ in measurements obtained at body $(\sim 1.79 \mathrm{~S} / \mathrm{m})$ and room $(\sim 1.45 \mathrm{~S} / \mathrm{m})$ temperature as revealed from Baumann and colleagues (1997), hence values at room temperature were revealed prior to regression and comparison of means analysis. Variability in CSF conductivity was discovered to be insignificantly explained by the weighted multiple regression model. Despite insignificant results, the boxplot in Fig. 8 allowed for a graphical representation of the large spread of values obtained using MREIT.

\section{Brain}

Differences in whole-brain conductivity values were not significantly predicted by the independent variables according to the weighted multiple regression analysis. Figure 9 reveals the large variation in data obtained for conductivity values of the whole-brain for each methodology, suggesting no one method generates a stable result for conductivity of the brain as a whole compartment.

\section{Grey Matter}

Variation in GM conductivity was not significantly explained by the weighted multiple regression model. However, a oneway ANOVA determined significant differences in GM conductivity according to method [Fig. 10; $F(3,62)=17.896$, $\mathrm{p}<.001]$, where results obtained with MREIT were significantly higher than DTI which were in turn significantly higher than EIT. Pathology further yielded significantly different conductivity results for GM [Fig. 11; F(4, 61) $=2.968, p=.026]$.

\section{White Matter}

A weighted multiple regression analysis revealed variation in isotropic WM conductivity was significantly explained by methodology, condition, frequency, pathology and age collectively $\left[\mathrm{R}^{2}(5,36)=.696, \mathrm{p}<.001\right]$, where values varied significantly according to method [Fig. $12 ; \mathrm{F}(4,99)=34.659$, $\mathrm{p}<.001]$, condition $[\mathrm{F}(2,101]=30.089, \mathrm{p}<.001]$, pathology $[\mathrm{F}(2,101)=34.437, \mathrm{p}<.001)$, temperature $[\mathrm{t}(102)=3.877$, $\mathrm{p}<.001]$ and frequency $[(\mathrm{r}(104)=-.362, \mathrm{p}=.001]$. Furthermore, pathology and age collectively explained a significant proportion of variation in WM conductivity measured perpendicularly $\left[\mathrm{R}^{2}(2,14)=.459, \mathrm{p}=.014\right]$ and 


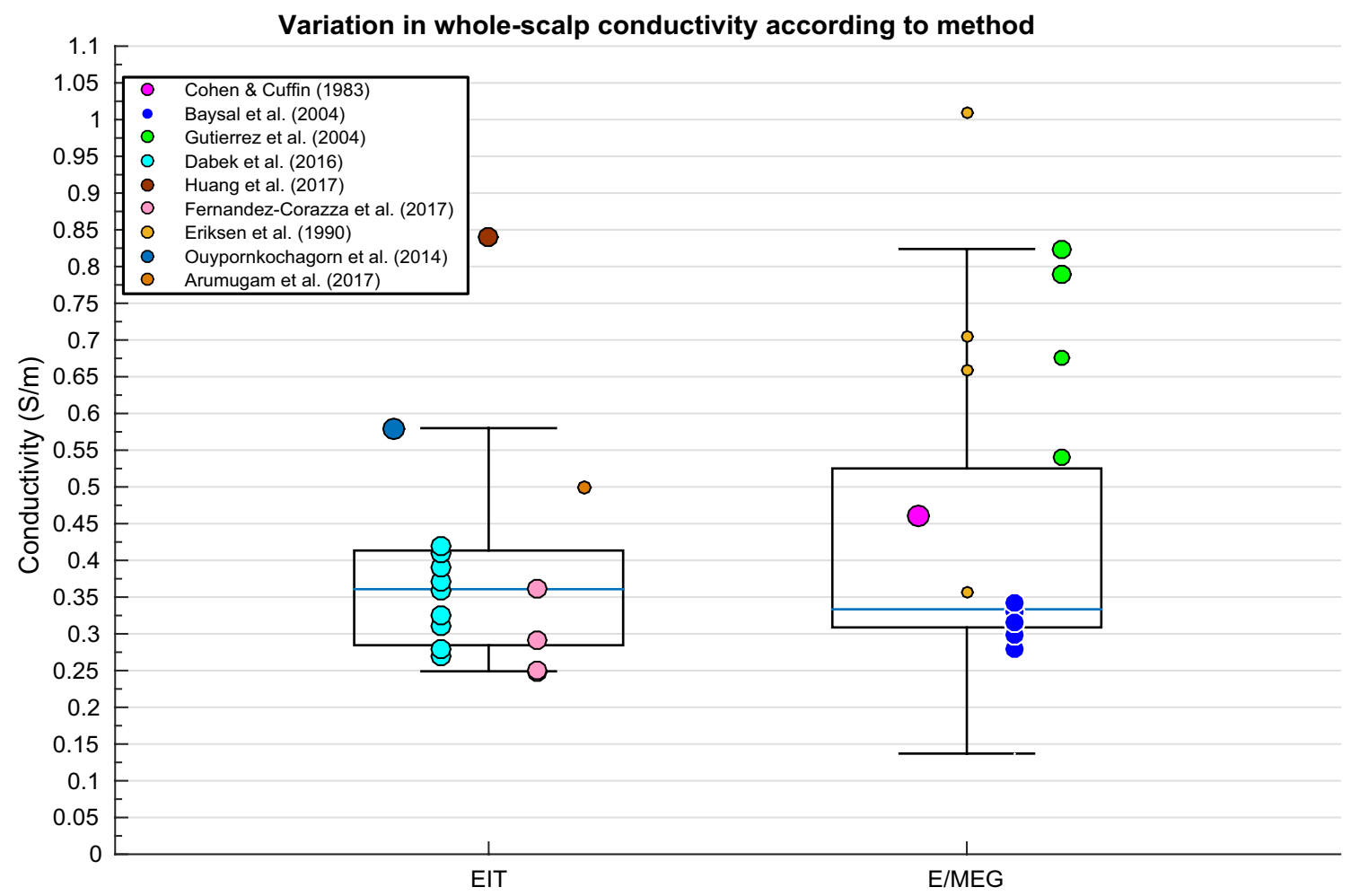

Fig. 4 Boxplot displaying inter-quartile range (box), medium (solid blue horizontal line), maximum and minimum (upper and lower whiskers respectively) of scalp conductivity according to method for each available paper. Size of data points indicates relative weight of value

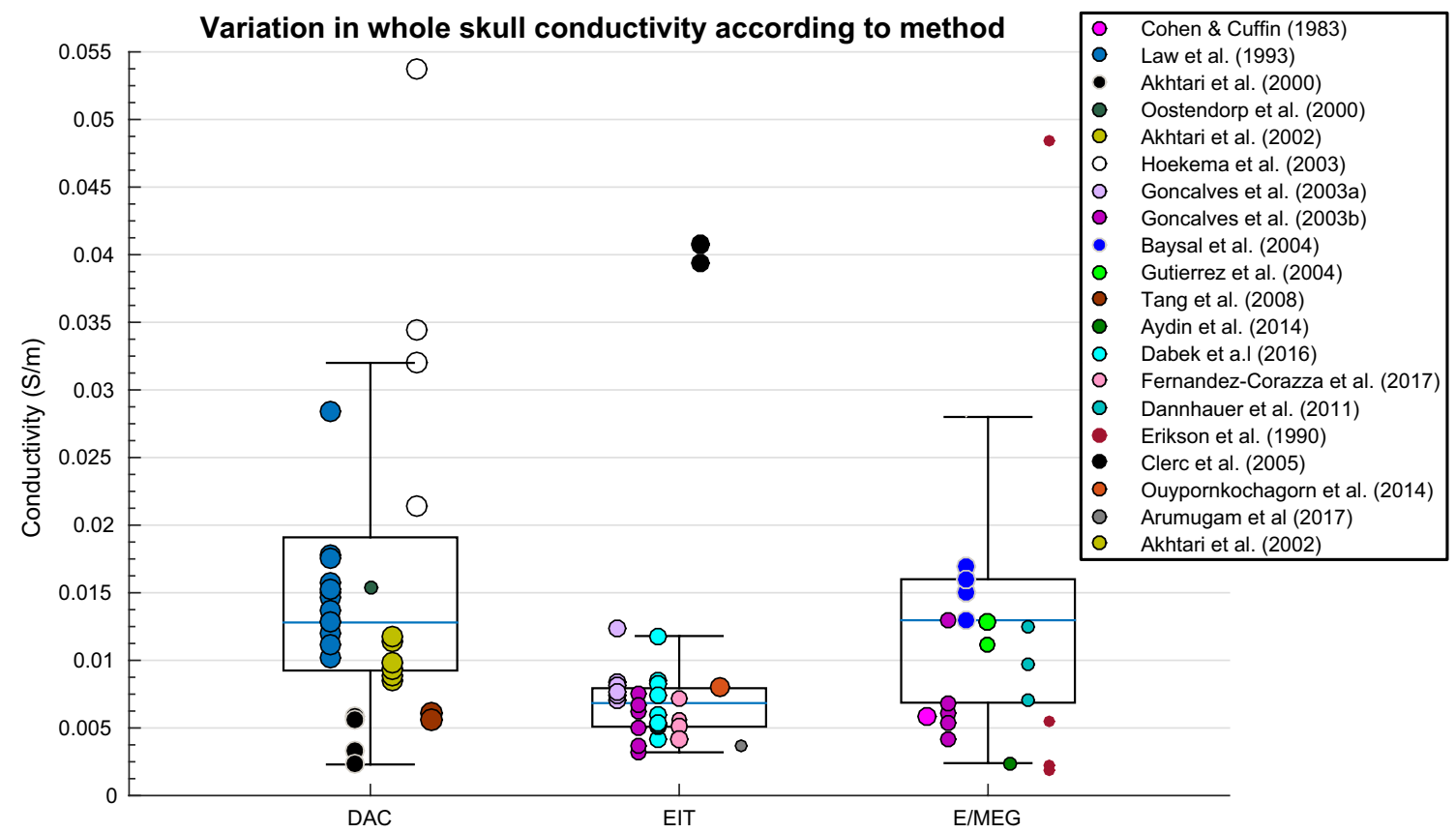

Fig. 5 Boxplot displaying variation in whole-skull conductivity according to method 


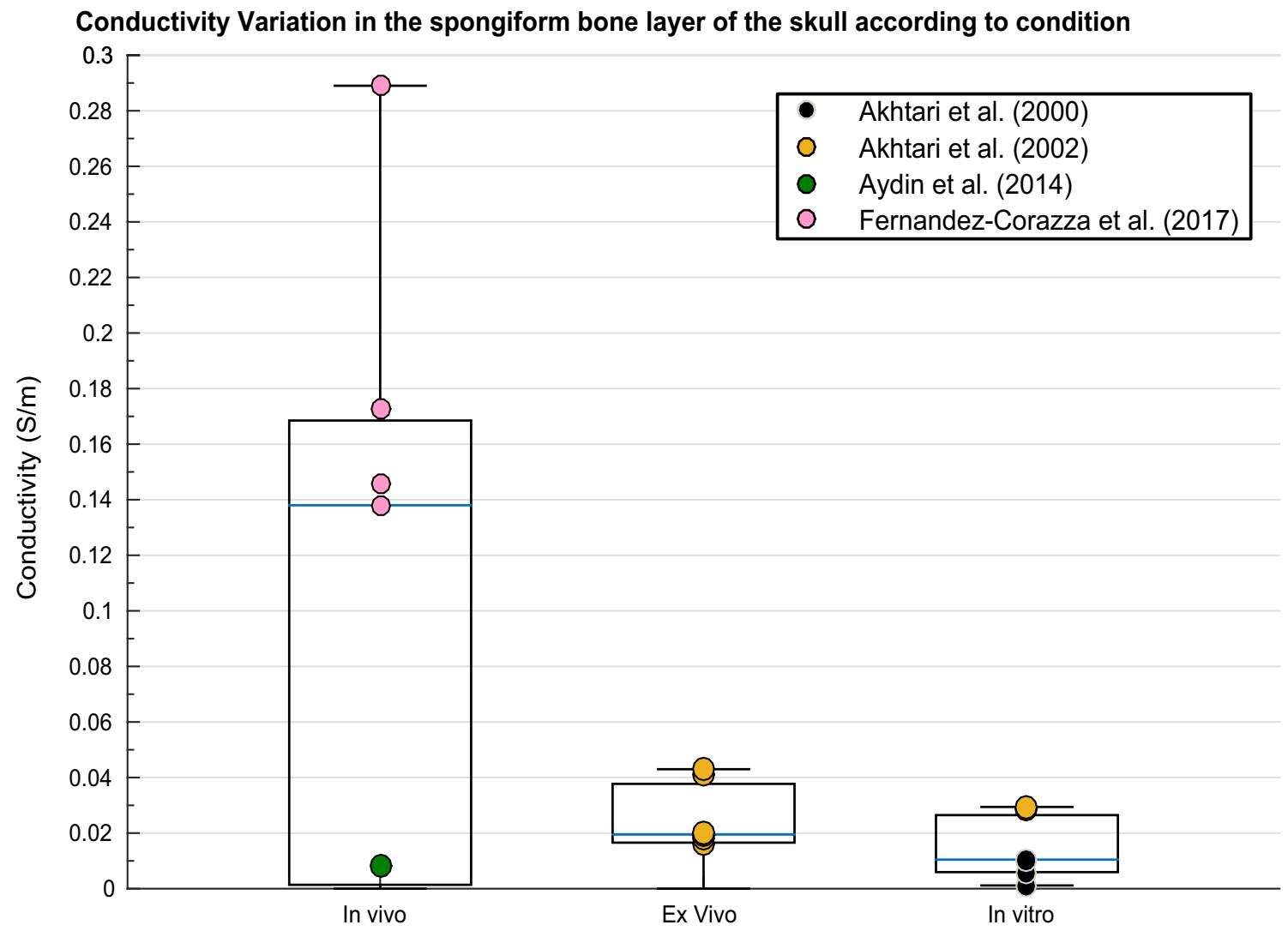

Fig. 6 Boxplot displaying variation in conductivity of the spongiform bone layer of the skull according to condition

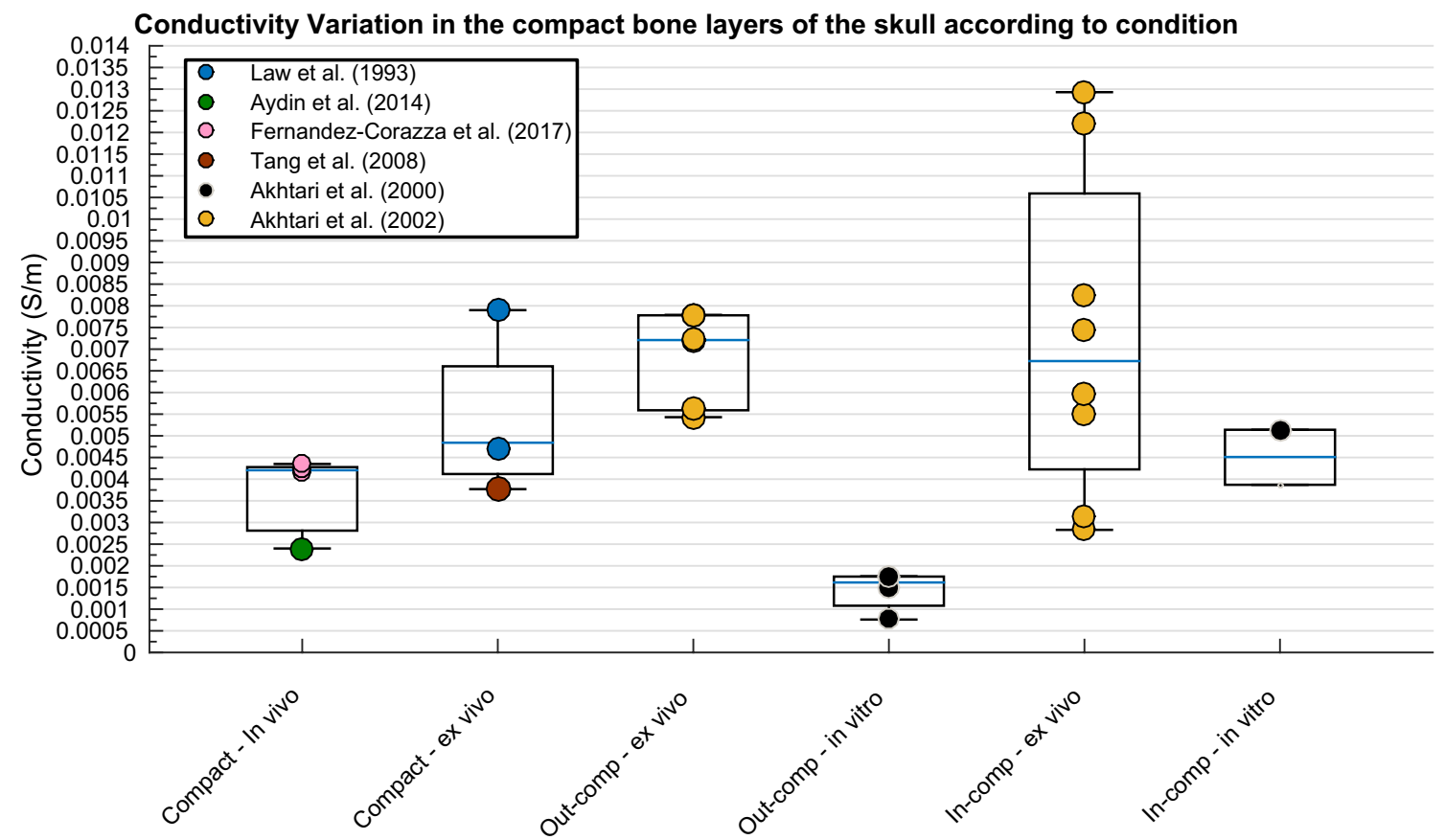

Fig. 7 Boxplot displaying variation in conductivity of the compact layers according to condition 


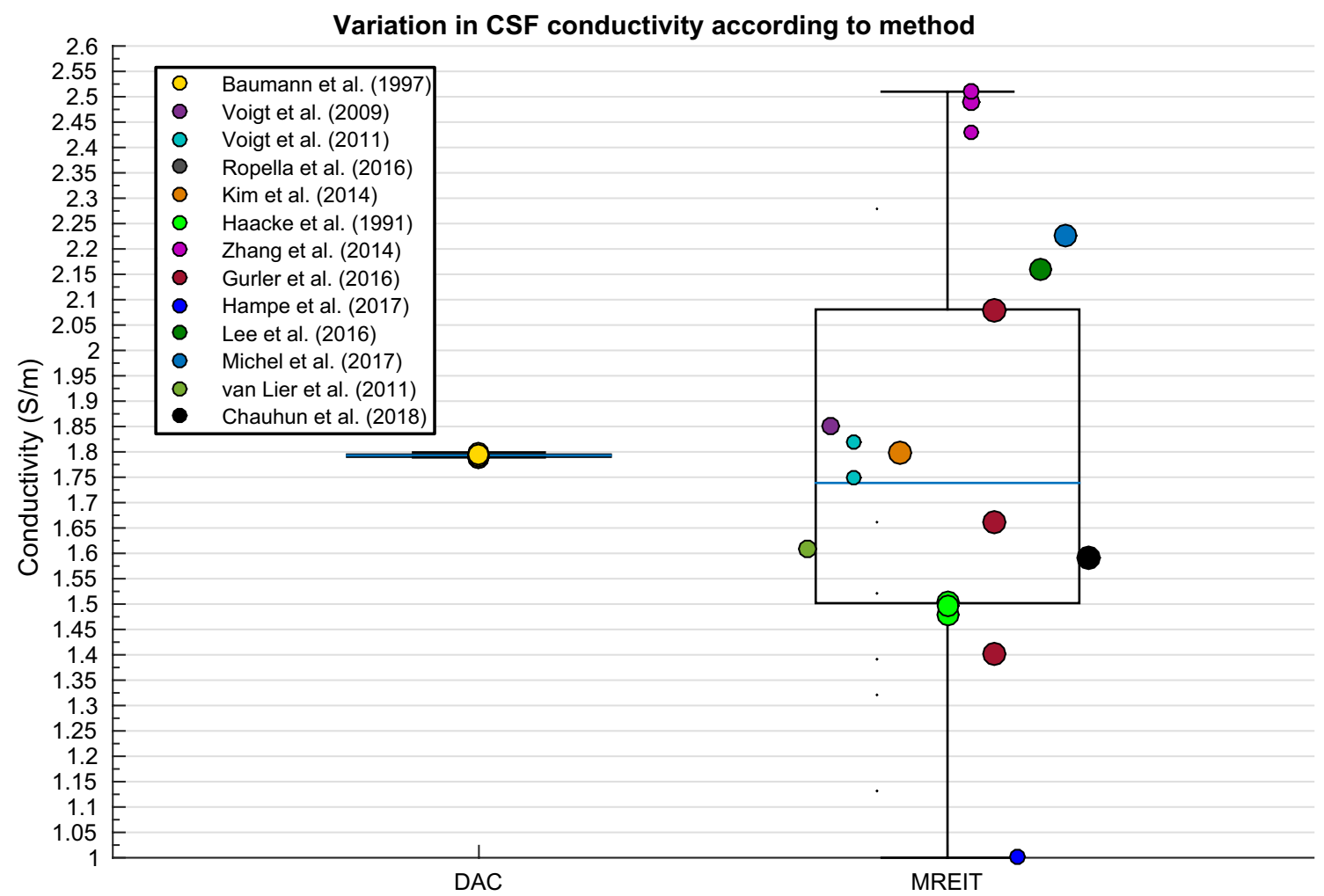

Fig. 8 Boxplot displaying variation in CSF conductivity depending on method

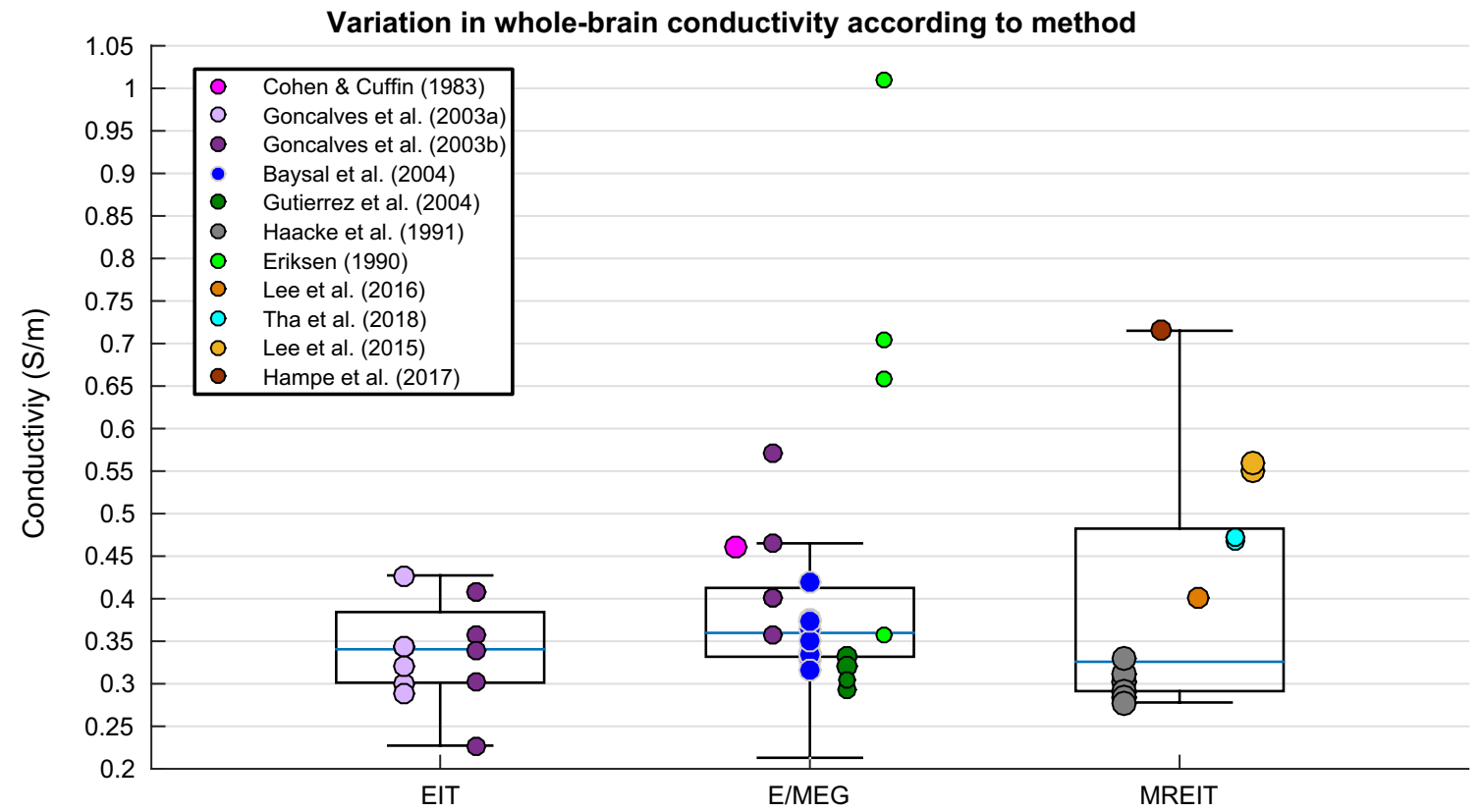

Fig. 9 Boxplot displaying variation in whole-brain conductivity depending on method

in parallel $\left[\mathrm{R}^{2}(2,14)=.677, \mathrm{p}<.001\right]$. Where perpendicular $\mathrm{WM}$ values varied according to condition $[\mathrm{F}(2$, $38)=36.828, \mathrm{p}<.001]$, temperature $[\mathrm{t}(39)=1.105, \mathrm{p}=.031]$ and participant age $[\mathrm{r}(41)=.638, \mathrm{p}=.006]$, whilst parallel WM measurements differed with condition $[\mathrm{F}(2,38)=9.78$, $\mathrm{p}<.001]$ and age $[\mathrm{r}(41)=.520, \mathrm{p}=.032]$. 


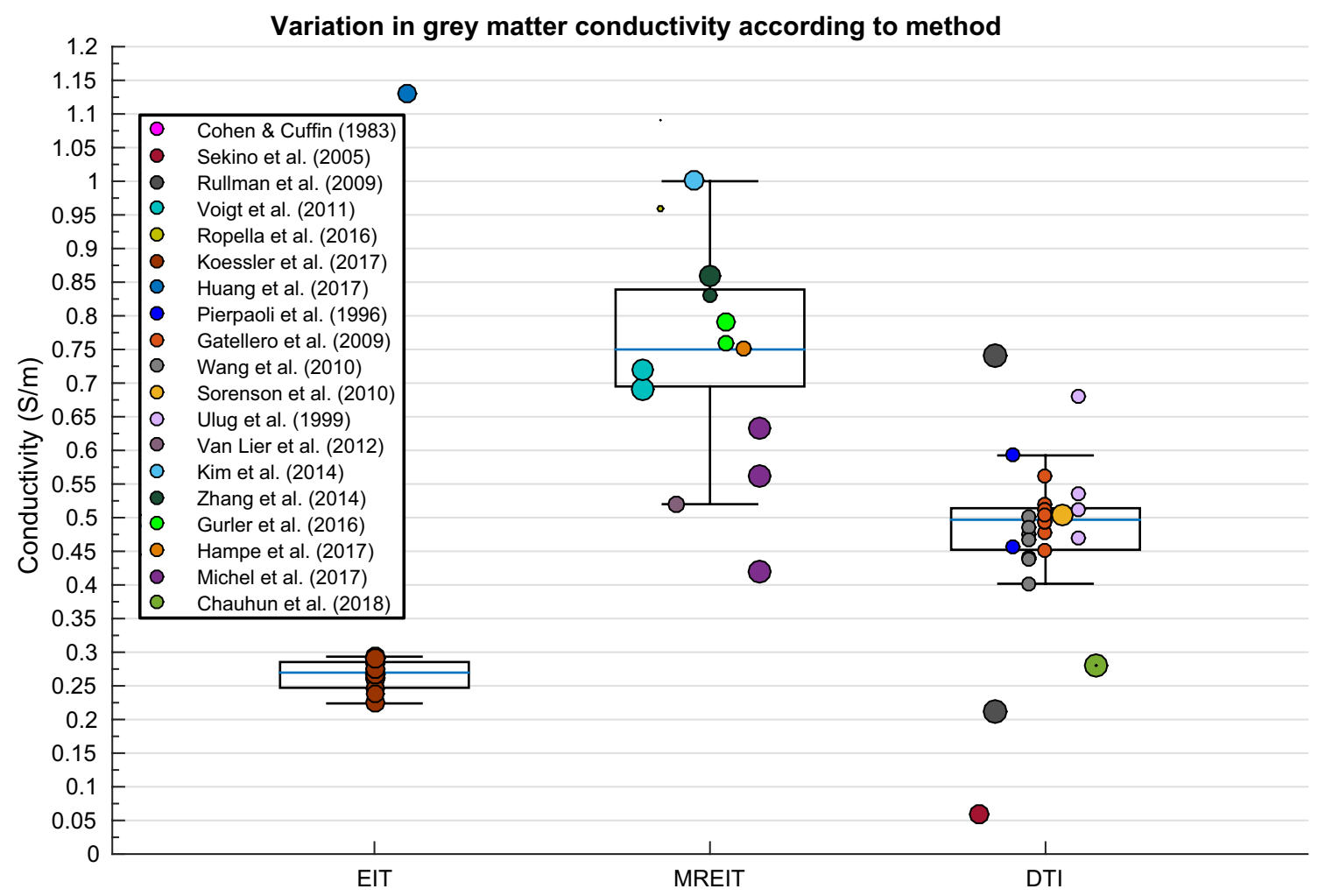

Fig. 10 Boxplot displaying variation in GM conductivity depending on method

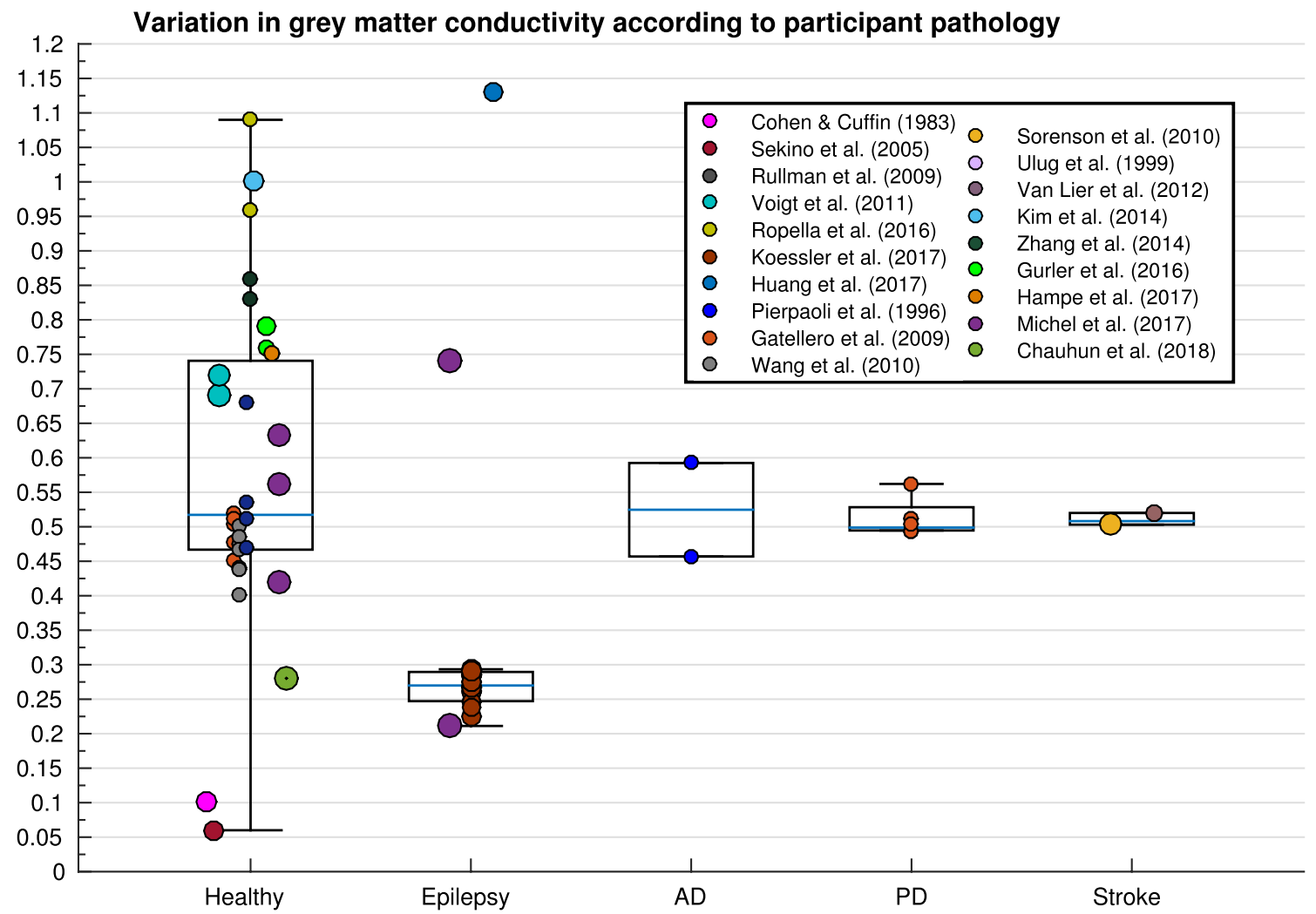

Fig. 11 Boxplot displaying variation in GM conductivity depending on pathology ( $A D$ Alzheimer's disease, $P D$ Parkinson's disease) 


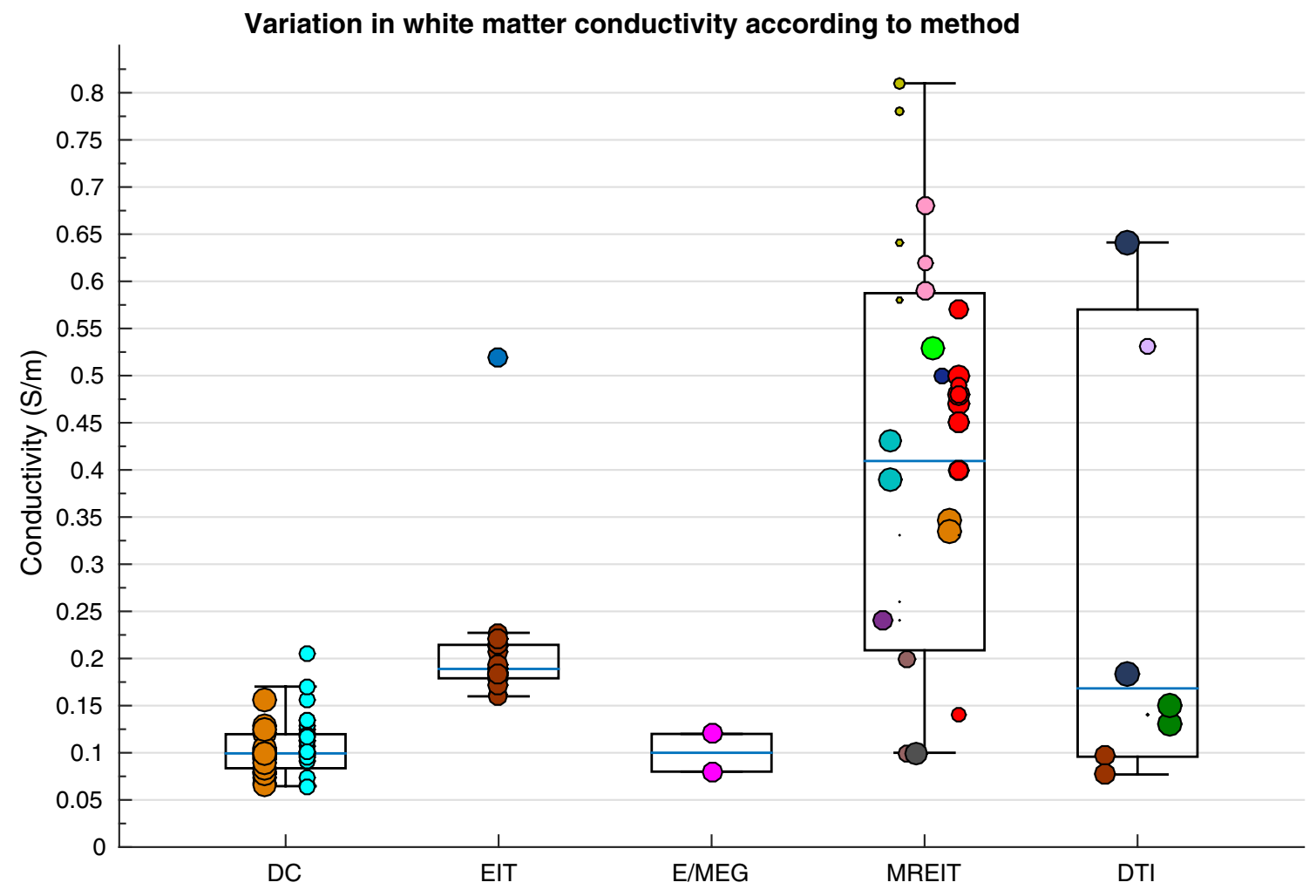

Fig. 12 Boxplot displaying variation in WM conductivity according to method

\section{Brain to Skull Conductivity Ratio (BSCR)}

Variation in BSCR calculations was significantly predicted by methodology, frequency, pathology and age collectively, in the weighted regression analysis $\left[\mathrm{R}^{2}(4,26)=.302\right.$, $\mathrm{p}=.046]$. Figure 13 displays the variation of BSCR according to method, although a comparison of means revealed no significant differences between employed technique. Additionally, BSCR values correlated positively with participant age [Fig. 14; $\mathrm{r}(51)=.376, \mathrm{p}=.014$ ].

\section{Discussion}

The current study systematically investigated variation in conductivity of 17 different head tissues as reported in 56 research papers, identified through a literature search of three relevant databases. The mean, standard deviation, minimum and maximum were calculated for each tissue type (Table 3 ). In addition, we computed the weighted average mean, which provided an optimum (and therefore suggested) value when conductivity is unable to be obtained on an individual basis. The weighted average mean and standard deviation (in $\mathrm{S} / \mathrm{m}$ ) for each tissue type were: $\mathrm{scalp}=0.41 \pm 0.18$, whole skull $=0.02 \pm 0.02$, spongiform skull layer $=0.048 \pm 0.07$, whole compact skull layer $=0.005 \pm 0.002$, outer compact $=0.005 \pm 0.003$, inner compact $=0.007 \pm 0.004$, $\mathrm{CSF}=1.71 \pm 0.3, \mathrm{GM}=0.47 \pm 0.24, \mathrm{WM}=0.22 \pm 0.17$, $\mathrm{WM}$ perpendicular $=0.12 \pm 0.05, \mathrm{WM}$ parallel $=0.12 \pm 0.09$, Blood $=0.57 \pm 0.11$ and $\mathrm{BSCR}=50.4 \pm 39$. The differences between values for each tissue were statistically tested against methodological and participant demographical variables to reveal significant predictors. Inadequate data was available for muscle, fat, blood, the epileptogenic zone, the dura layer, the cerebellum and brain lesions to carry out a multiple regression. Collectively, the independent variables (related to both methodology and demographics) insignificantly explained variation in the scalp, the compact layers of the skull, cerebrospinal fluid, the whole-brain and grey matter. In contrast, variation in whole-skull conductivity could significantly be explained by all the IV's collectively, where values were revealed to specifically differ significantly depending on method. Variation in the conductivity of the spongiform bone layer of the skull was significantly predicted by condition, frequency, pathology and age, where values were significantly different according to condition and temperature. Despite insignificant results for regression analysis, GM significantly differed depending on method and pathology. Variation in isotropic WM electrical conductivity was significantly predicted by methodology, condition, frequency, pathology and age collectively in the regression model, where values significantly diverged according to 


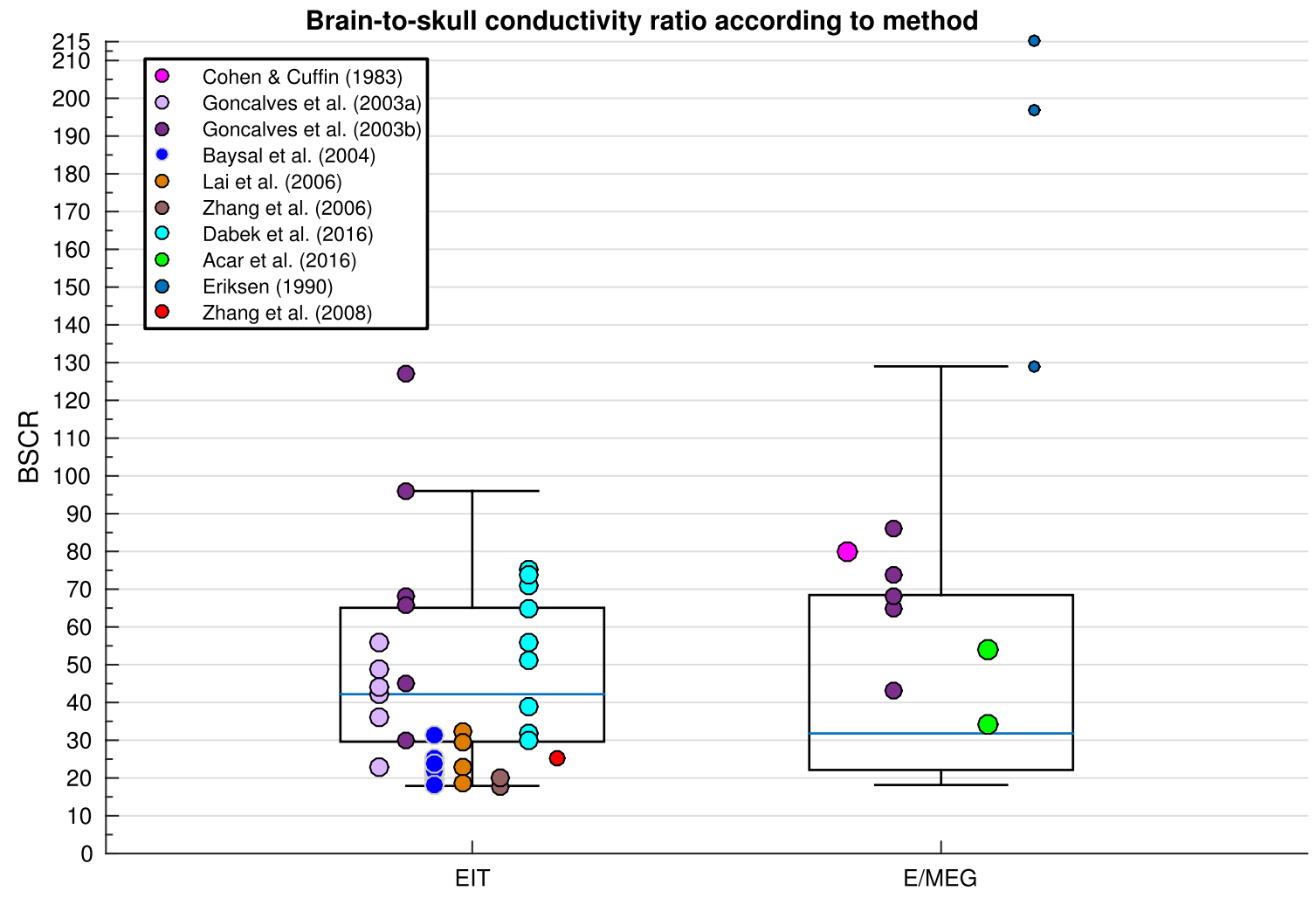

Fig. 13 Boxplot displaying variation in BSCR depending on method

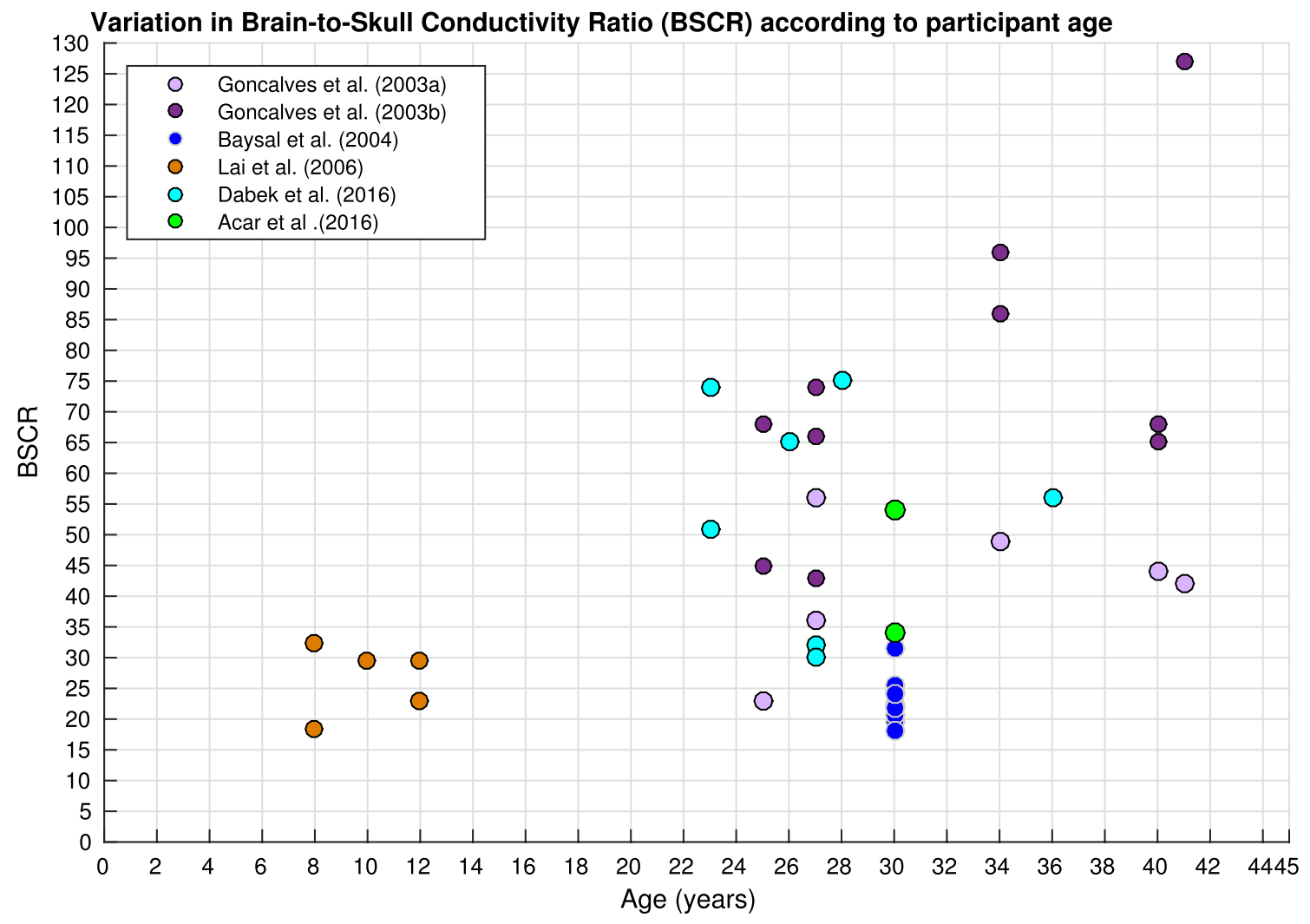

Fig. 14 Scatter diagram displaying BSCR as a function of participant's age 
method, condition, pathology, temperature and frequency. A significant proportion of variation in WM conductivity measured perpendicularly and in parallel was further explained by pathology and age collectively. Specifically, perpendicular WM values differed with condition, temperature and age, whilst parallel WM measurements fluctuated with condition and age. Lastly, the meta-regression revealed the BSCR could be significantly attributed to variation in methodology, frequency, pathology and age collectively, revealing a positive correlation between the ratio and participant's age.

\section{Data Exclusions}

Explanations for the presence of outliers and reasons for any data exclusion in the current meta-analysis are further discussed. Firstly, data acquired at frequencies higher than $1000 \mathrm{~Hz}$ were removed, as these conditions were deemed unnatural, considering the bandwidth of most neuronal signals is $1 \mathrm{~Hz}-1 \mathrm{kHz}$, with resonant frequencies of $<100 \mathrm{~Hz}$ for the brain (Groppe et al. 2013) and $<1000 \mathrm{~Hz}$ for the skull (Håkansson et al. 1994). Conductivity results obtained from Baysal and Haueisen (2004a, b) employing a conventional least-squares estimator (LSEE) on E/MEG data were further revealed from the weighting algorithm, as stated by the authors as being "unrealistic [negative resistivities] and unstable" Baysal and Haueisen (2004a, b). These unstable results were evident from the large standard deviation percentage $(>5000 \%$ for the scalp, $>200 \%$ for the skull and $>240 \%$ for the brain). The authors suggested such inaccuracies occurred from the use of LSEE linearization in a highly non-linear problem, and hence were omitted from their own analysis. Furthermore, skull conductivity values reported by (Hoekema et al. 2003) were elevated approximately 10-fold, suggested to be "as expected [due to measurements] in non-physiological circumstances" (namely, saline-coated cadaver), however were not excluded from the current analysis as methodology was in line with previous cadaver studies and therefore should yield similar results. Huang and colleagues (2017) yielded significant outliers for the scalp, skull, whole-brain, GM and WM, where median optimal conductivity obtained by fitting model outputs (from literature) to recordings. These deviations may be explained by the use of an optimisation EIT approach where "best-fit" values were free to compensate for all inaccurate/simplified sources, tissue segmentation errors, changes in electrode location, etc., and therefore cannot reflect "true" conductivity values. Outliers were additionally revealed for the spongiform (but not compact) skull layer by Fernández-Corazza and colleagues (2017), which employed boundary EIT (bEIT) for reconstructing the electrical conductivity for a subset of the regional tissue parameters. The authors acknowledged low sensitivity of bEIT to spongiform variations, due to the relatively small proportion of spongiform to head volume and concluded such approximations may be difficult for unbiased bEIT estimators but remain valid for compact bone estimates. Substantial outliers were further exposed for CSF, GM and WM (as well as subsequent EMA whole-brain calculations) in Ropella and Noll's (2017) paper, which employed MREIT and revealed standard deviations of $80.2-518.8 \%$. The validity and reliability of MREIT as a method is later discussed. The Quality Analysis in the current paper attributing a confidence weighting to each value, is deemed acceptable to consider the large standard deviations leading to outliers. All outliers were therefore included in order to fully account for and explore reasons for variation in values.

\section{Scalp}

Additional deviations, although not classified as outliers, were revealed from (Gutierrez et al. 2004) for the scalp, where maximum likelihood estimation (MLE) was used to estimate the electrical conductivity from E/MEG measurements. Such results may be due, in part, to the necessity of accurate source location and head geometry knowledge to avoid estimation bias. The authors suggest the use of a Bayesian approach, which permits incorporating a priori information on conductivity distribution to reduce bias. Realistic measurements (excluding outliers and deviations, as discussed) of scalp conductivity, for example, ranged between 0.25 and $0.435 \mathrm{~S} / \mathrm{m}$, which could not be attributed to any of the IV's. Such variation can be relevant in source localisation based on E/MEG. In particular, dipole sources close to measurement electrodes are sensitive to scalp conductivity (Gençer and Acar 2004; Goncalves et al. $2003 \mathrm{a}, \mathrm{b}, \mathrm{c})$. These results coupled with those of the current meta-analysis indicate that assuming scalp conductivity from the literature is not only inaccurate but can lead to E/ MEG source mislocalisation. These errors do not appear to be explainable by anything other than individual variability and hence personalised models of scalp conductivity should be considered to improve E/MEG activity localisation.

\section{Skull}

According to the meta-regression, variation in whole-skull conductivity can be accounted for by differences in all of the independent variables (methodology, condition, temperature, frequency, pathology and age), with specific differences between methodology and condition. Such significant results, however, may be due to overfitting of the data and meta-regression parameters employed. Future research could utilise machine learning techniques in order to refine the regression analysis and determine the most influential variables. This analysis was beyond the scope of the current 
paper and ideally would require more data which is not presently available.

Values obtained from excised tissue in non-physiological circumstances after undergoing various processing, may change the electrolyte concentration and therefore skull conductivity (Akhtari et al. 2002). Considering the contrast between a saline-soaked processed cadaver and live skulls that remain in natural conditions between the scalp and meninges, differences in conductivity values post-mortem should be expected. Early research determined conductivity of rat femurs to decrease by a factor of 2.5-3 from liveto-50 h post-mortem (Kosterich et al. 1983, 1984). This was further corroborated in the human skull, indicating a scaling factor of 2.5-4 from live-to-post-mortem (Wendel et al. 2006). These results are validated by Rush and Driscoll's mathematical model stating the skull conductivities dependency on the fluid (i.e. saline vs naturally positioned between meninges, surrounded by blood/CSF, etc.) permeating it (Rush and Driscoll 1969), suggesting, a live skull to have higher conductivity than a saline-soaked (with conductivity $1 \mathrm{~S} / \mathrm{m}$ ) cadaver skull. It is consequently unsurprising in vitro values of skull conductivity differ from in vivo values-predicting increased conductivity in vivo (Akhtari et al. 2000; Law 1993). The results from the previous literature coupled with the current meta-analysis, therefore indicate skull conductivity should be measured in in vivo (i.e. at body temperature, within the live head, etc.) in order to avoid bias and increase reliability.

Within in vivo reports, despite all under similar conditions, skull conductivity values obtained using E/MEG appeared to be elevated compared to those employing EIT. These results may be explained by the use of statistically constrained estimating algorithms for E/MEG measurements, for which conductivity is optimally estimated from E/MEG arrays acquired during electrical nerve stimulation. Furthermore, whole-skull conductivity was found to vary as a function of frequency, although the nature of the relationship was unclear. Previous literature has examined skull conductivity in wider frequencies, revealing a positive relationship, especially at frequencies higher than $10 \mathrm{kHz}$ (Gabriel et al. 1996b; Tang et al. 2008), and suggest skull conductivity may exponentially increase at high frequencies. Within the relevant range for brain activity, skull conductivity increased by $\sim 6.7 \%$ from 11 to $127 \mathrm{~Hz}$ (Dabek et al. 2016) and 13\% from 10 to $90 \mathrm{~Hz}$ (Akhtari et al. 2002), of which the authors developed a non-linear model for frequency dependence of different skull layers (Akhtari et al. 2003). Variation as a function of frequency may be expected due to interactions between mobile electrolytes (i.e. sodium and chloride) and relatively immobile molecules (i.e. proteins and blood components) which affect the relaxation rate of conductivity dependent on the currents frequency (Akhtari et al. 2002; Latif et al. (2010). Importantly, this frequency dependence has been implicated in causing the volume conductor to act as a low pass filter, which potentially contributes to E/MEG forward solutions errors and may therefore reduce accuracy in E/MEG source localisation.

Furthermore, results exploring differences according to age in the BSCR (see Fig. 14) have implications for skull conductivity variation. Research has indicated skull, rather than brain, conductivity plays a larger role in BSCR values (Goncalves et al. 2003a, b, c), consequently suggesting skull conductivity varies with age. These results are discussed further in Sect. 4.3.1 (layered skull conductivity) and 4.6 (BSCR).

\section{Layered skull}

The majority of previous studies simplify the skull as a homogeneous layer, not accounting for differences in conductivity between the compact (upper, lower) and spongiform layers of the skull. Distinct conductivities for the three layers of the skull has been previously indicated (Akhtari et al. 2000; Akhtari et al. 2002; Fernández-Corazza et al. 2017; Tang et al. 2008), as supported by the current metaanalysis (see Fig. 2). This is unsurprising considering the higher prevalence of fluid filled pores and cavities in spongiform (and hence higher conductivity) compared to compact bone. Importantly, neglecting inhomogeneous estimations for a tri-layer skull has yielded significant errors in source localisation irrespective of model parameters (Dannhauer et al. 2011; Haueisen et al. 1999; Haueisen et al. 2002; Ollikainen et al. 1999; Pohlmeier et al. 1997). These authors have thus concluded realistic modelling of the skull layers to be necessary for accurate E/MEG source localisation. The current meta-analysis, however, revealed that variations exist between individuals even whilst considering a tri-layer skull. Variation in the electrical conductivity of the spongiform skull layer was revealed to be significant and attributed to deviations in condition, temperature, frequency, pathology and age. Suggesting true values for conductivity of the spongiform layer will not only depend on methodological parameters but also individual demographics. Likewise, although the compact layers of the skull were insignificantly predicted by any of the parameters, large variation was still evident. This further elucidates the hypothesis that conductivity values fluctuate between individuals and support the suggestion for personalised models of skull conductivity.

Interestingly, a relationship with age was to be expected, regardless of homogeneity, considering the presence of the fontanels and open sutures (un-ossified bone filled with cartilage, chondroid and vascular tissue) which may remain unfused for several years (Hansman 1966). Firstly, bone formation (remodelling) from direct laying down or cartilage, known as ossification, remains approximately $50 \%$ complete at birth, not reaching $100 \%$ until age 20 . This indicates a 
higher proportion of cartilage and "soft" (trabecular) bone, consisting of higher water and less lipid content (Silau et al. 1995), in the neonatal skull, which linearly decreases until fully ossified (Christie 1949). Furthermore, neonatal brain growth occurs at a rate higher than bone ossification, resulting in four fontanelles between the skull plates. The fontanelles, although relatively small at birth, become larger within the first few months (up to $3 \mathrm{~cm}$ ) until closing at approximately 18 months old (Hansman 1966). As well as inter-subject variability, the size and width of fontanelles significantly vary in children with central nervous system pathology. Moreover, unclosed sutures in an infant skull, although present in an adult skull, are wider near the fontanelles, and may not close for several years in healthy children (Erasmie and Ringertz 1976). In addition to the fontanelles, the sutures close at various times of development. The frontal suture, for example, normally fuses between 3 and 9 months old (Vu et al. 2001), whereas the sphenofrontal suture (lying between the sphenoid bone and posterior horizontal orbital plates) and sagittal suture (connecting the two parietal bones) typically close by age 15 and 22 years, respectively. Moreover, the squamosal sutures (connecting the temporal squama and parietal bone), do not fully close until 60 years of age (Vijay Kumar et al. 2012). Lastly, the skull thickness increases with age, from $2-3 \mathrm{~mm}$ at birth to 3-6 $\mathrm{mm}$ during early adulthood (Despotovic et al. 2013; Hansman 1966). This increase however is non-linear, slowing down towards 3 years of age, and is non-uniformly distributed throughout the skull, with higher thickness in occipital than frontal and parietal regions (Li et al. 2015).

Taken together, the structural differences between the neonatal and adult skull elucidate differences in skull conductivity to be expected. As such, previous studies have revealed higher skull conductivity for infants compared to adults, (Gibson et al. 2000; Pant et al. 2011) and an inverse correlation between skull conductivity and thickness with increasing age (Gibson et al. 2000). The inner and outer compact layers are thought to become thicker, whilst the spongiform layer becomes thinner with age, hence decreasing whole-skull conductivity. Additionally, paediatric skull tissue ordinarily contains greater quantities of ions and water, compared to calcified cranial bones of adults, hence higher conductivity may be expected (Schönborn et al. 1998). Further support from animal studies have revealed skull conductivity to decrease with age, for example, in the rat (Peyman et al. 2001), pig (Peyman et al. 2007) and cow (Schmid and Überbacher 2005). Although this expectation was not confirmed in the current meta-analysis, for accurate source localisation, skull conductivity variations with age should be taken into consideration. For example, Lew and colleagues assessed the effect of sutures and fontanels on E/ MEG source analysis, where omission produced a maximum position error of $3.6 \mathrm{~mm}$ and $0.6 \mathrm{~mm}$ for tangentially- and radially-oriented sources, respectively (Lew et al. 2013). Elevated EEG localisation errors with respect to MEG were further replicated in simulation studies (Flemming et al. 2005), suggesting an advantage in employing MEG for developmental studies. The literature however suggests both modalities require individualised, or in the least an infant-specific volume conductor model to accommodate for relevant developmental changes (Bystron et al. 2008; Rakic 2006; Song et al. 2013).

Following from this, development of the human skull does not cease after infancy, but continues to undergo remodelling, microstructural, density and histological changes until death, further impacting conductivity. Firstly, total cranial thickness has been observed to increase with age (Todd 1924) notably related to increase in diploë thickness (Hatipoglu et al. 2008; Sabancıoğulları et al. 2012 which in one study was accompanied with inner and outer compact thinning (Skrzat et al. 2004). An increase in diploë (and hence spongiform bone) thickness would suggest conductivity of the skull to increase with age, as revealed by (Tang et al. 2008). Recently, Antonakakis and colleagues (2018) revealed a trend between participant age and skull conductivity but noted the small sample size $(n=15)$ and large inter-subject variability rendered robust conclusions difficult and inadequate. Further results have also been inconsistent, finding no such relationship between skull thickness and age (Ishida and Dodo, 1990; Lynnerup 2001; Lynnerup et al. 2005; Pensler and McCarthy 1985; Sullivan and Smith, 1989). The presence of suture lines, not limited to infants, was furthermore shown to increase conductivity of the skull sample, by providing a path of high conductance (Law 1993; Tang et al. 2008). Additionally, the percentage of spongiform bone within the skull was positively correlated with skull conductivity (Tang et al. 2008), whilst, skull thickness, which is non-uniform within and between individuals (Lynnerup 2001; Lynnerup et al. 2005), was inversely correlated with scalp potentials (Chauveau et al. 2004). One paper, for example, revealed that a $20 \%$ and $40 \%$ decrease in skull thickness resulted in a 5-10\% and 20-25\% decrease in conductivity, respectively (Lai et al. 2005). Insufficient results were available to analyse the influence of sutures or skull thickness in the current review; however, the discussed structural deviations further illuminate the importance of employing individualised models of head conductivity.

The influence of skull conductivity and segmentation inaccuracies has been explored extensively, revealing overwhelming source localisation errors for EEG (Lanfer et al. 2012; Montes-Restrepo et al. 2014; Wolters et al. 2006) and MEG (Cho et al. 2015; Lau et al. 2016) of up to $2 \mathrm{~cm}$. E/ MEG source analysis can importantly be utilised during presurgical epilepsy diagnoses, together with MRI data. Aydin and colleagues (2017) recently developed a multimodal technique, of which they emphasised the importance 
of individualised high-resolution finite element head models with WM anisotropy modelled from DTI and individually calibrated skull conductivity, alongside combined E/ MEG and MRI information. Importantly, they note creating such realistic head models may not always be feasible, and therefore recommend, at minimum, skull conductivity to be individually adjusted to improve combined E/MEG source analysis. Variations in skull conductivity have been found to impact transcranial electric stimulation focality and dose (Santos et al. 2016; Schmidt et al. 2015; Wenger et al. 2015), with one study revealing an error of $8 \%$ in dose (FernándezCorazza et al. 2017). Such inaccuracies are clinically relevant, particularly regarding source estimation for refractory epilepsy (Brodbeck et al. 2011) and determining electrical current dose required for treatment of epilepsy (Berényi et al. 2012; Liebetanz et al. 2006), depression (Kalu et al. 2012) and other psychiatric disorders (Brunoni et al. 2013). Of note, Dannhauer and colleagues (2011) investigated variations in layered skull structures for EEG forward modelling and revealed an inhomogenous but not isotropic modelling to be of most importance. For optimum skull modelling, they recommended assigning each skull voxel to a tissue type (compact or spongiform bone) with individually estimated or measured conductivity values. To optimise source localisation accuracy, we therefore recommend employing personalised models of skull conductivity to sufficiently consider individual variability.

\section{CSF}

The results of the current meta-analysis are in line with previous report from Baumann and colleagues (1997), displaying significant variation in CSF conductivity dependent on temperature. They revealed $23 \%$ higher conductivity at body $\left(37^{\circ} \mathrm{C}\right)$, approximately $1.79 \mathrm{~S} / \mathrm{m}$, than room $\left(25^{\circ} \mathrm{C}\right)$ temperature, which corresponded to the temperature coefficient of $2 \%$ per $0.1 \mathrm{ml}$ of potassium chloride (comparable to CSF conductivity and ion concentration (Fishman, 1992; McGale et al. 1977; Wu et al. 1991). The result from Baumann et al. (1997) study is frequently considered as a reference value for CSF conductivity. However, the current meta-analysis has revealed large variation in measurements, potentially suggesting instability in CSF conductivity between individuals. The majority of such variation stems from values obtained using MREIT. One such study, (Ropella and Noll 2017) reported values with standard deviations up to $518 \%$ of the mean, which indicates instability in the methodology. The combination of large standard deviations within and between studies potentially call into question the validity of MREIT for measuring conductivity of the human head - this is further discussed in the grey matter subsection (4.5.1). Further deviating from Baumann's approximation (1.79 $\mathrm{S} / \mathrm{m}$ ), (Cohen and Cuffin 1983b) reported a considerably lower conductivity for CSF $(1.39 \mathrm{~S} / \mathrm{m})$. These results may be explained by their use of optimum estimation, rather than direct measurements; where conductivity was adjusted so the maximum potential in a theoretical EEG map matched the experimental equivalent. Considering the variation due to methodological error, CSF appears to be relatively stable between individuals, with an average conductivity converging around Baumann's results. Despite this, Fig. 8 suggests CSF conductivity ranges between $\sim 1.5$ and $2 \mathrm{~S} / \mathrm{m}$, elucidating the need for individualised measurements. Deviation in CSF conductivity in that range however, may not significantly affect E/MEG forward and inverse modelling solutions. Future work could therefore employ a sensitivity analysis to explore the influence CSF conductivity has on electromagnetic source localisation and determine the necessity of individualised models.

\section{Whole-Brain}

The meta-regression failed to explain variation in conductivity of the brain as a homogeneous layer, however values were revealed to significantly vary according to the employed method. Despite these significant results, variation between acquisition techniques are minimal, where large variations within each method remain evident. These variations further support the suggestion that individual values of conductivity should be obtained. However, assuming homogeneous conductivity over the whole brain is generally considered a vast oversimplification and highly inaccurate. Such an assumption fails to consider differences between GM and WM conductivity, as well as structural variation of GM/WM proportion in the brain. Early research determined GM to contain higher proportions of water than WM (Stewart-Wallace 1939), demonstrating expected higher electrical conductivity for GM compared to WM. Additionally, extensive literature has shown GM and WM volume to vary with development (Giorgio et al. 2010; Groeschel et al. 2010; Miller et al. 1980) and pathology; i.e. multiple sclerosis (Sastre-Garriga et al. 2005), Alzheimer's Disease (Salat et al. 1999), schizophrenia (Douaud et al. 2007), attention deficit-hyperactivity disorder (McAlonan et al. 2007), among others. These observations further support the use of individualised models of head volume and conductivity profiles for the most accurate representation of the human head.

\section{Grey Matter}

The questionable validity of MREIT for estimating conductivity was further reinforced considering the number of outliers (Ropella and Noll 2017), coupled with elevated values (Voigt et al. 2011) for GM conductivity acquired with MREIT. Measurements utilising MREIT were over twice those from EIT and approximately one and a half times those 
of DTI values. Significant variation in GM conductivity may be further explained by increased DTI values relative to EIT. Firstly, the Tuch et al. (1999) model derived the conductivity tensor from the water diffusion tensor through differential effective medium approximation (EMA), which uses an electromagnetic depolarisation factor to consider the impact of cell geometry to overall conductivity. This depolarisation factor was originally developed for WM structure, consisting of myelinated pyramidal cells, and therefore may not be completely translational to GM. Tuch and colleagues' later paper (2001a, b), however, utilised the EMA method to show there exists a strong linear relationship between the conductivity and diffusion tensors, regardless of tissue type. They generated a conductivity tensor image, where conductivity could be assigned to three groups: GM, WM parallel or WM perpendicular to the fibre tract. Their results indicate the EMA method is appropriate for GM conductivity estimation, despite having lower anisotropy than WM. The established linear relationship mapping the diffusion to electrical conductivity tensor employed (Tuch et al. 1999; Tuch et al. $2001 \mathrm{a}, \mathrm{b})$ has been further validated in a silk yarn phantom (Oh et al. 2006). However, (Rullmann et al. 2009) detected the use of Tuch's scaling factor would have generated values 3.5 times greater than isotropic values (taken from (Ramon et al. 2006)). For this reason, they chose to employ a volume constraint approach with scaling factor 0.21 (compared to 0.844) which minimised differences between isotropic and anisotropic EEG forward modelling in their study. Similarly, (Sekino et al. 2005) estimated the effective GM conductivity from only the fast diffusion component (attributed to extracellular fluid), rather than both the fast and slow (attributed to intracellular fluid) components. The produced conductivity maps were therefore not simply linearly scaled diffusion maps, and hence may explain their considerable low GM conductivity measurement $(0.06 \mathrm{~S} / \mathrm{m})$. Importantly, the deviations in GM conductivity dependent on the chosen diffusion tensor method are acknowledged, emphasising the non-trivial nature of relating the diffusion and electrical conductivity tensors. Future studies should examine this relationship, in order to accurately determine a realistic scaling factor to improve conductivity tensor estimations.

Additionally, Rullmann and colleagues (2009) results were limited to one paediatric participant with epilepsy, of which his age may have influenced the increased brain conductivity. Higher conductivities in paediatric brains are perhaps expected due to the general abundance of water in GM (Dobbing and Sands 1973). The current meta-analysis failed to find a significant correlation between GM conductivity and age, however, considering normal GM development and the frequently observed decrease in mean diffusivity of GM with age (Pal et al. 2011), further research may expose such a relationship.
In line with this observation, participant pathology significantly affected GM conductivity, but was not a significant predictor in the regression model. Large variation can be seen within and between different participant pathologies (Fig. 11), but no clear conclusion could be made. This is perhaps due to the limited number of values available for each classified pathology, reducing the statistical power. In tumours, for example, firstly, all values may not have been made from healthy tissues (due to the diffusing nature of malignant tumours), but also an increased conductivity in "healthy" GM tissue may also be a consequence of tumour cysts increasing the water/CSF concentration in nearby tissues. Previous literature has indicated abnormalities in GM volume, structure, myelination and topography in Multiple Sclerosis, Parkinson's Disease, Alzheimer's Disease and other forms of dementia (Compta et al. 2012; Frisoni et al. 2007; Geurts and Barkhof 2008), as well as abnormalities in psychiatric and developmental disorders (Greimel et al. 2013; Job et al. 2005; Wise et al. 2017). It is therefore unsurprising that GM conductivity varied with participant pathology. Due to the unknown nature of this variation with disease and age, the use of individualised models of head conductivity that are inhomogeneous and anisotropic are especially essential for electromagnetic source imaging (Birot et al. 2014). Increasing the feasibility and accessibility of this could be explored with further research involving DTI parameters.

\section{White Matter}

The current meta-analysis failed to explain variation in anisotropic WM conductivity, however this may be due to the limited sample size available for analysis. The crucial consideration of anisotropic conductivities has been more recently determined, where neglecting WM anisotropy produced EEG-localisation errors of $\sim 1.6-5.1 \mathrm{~mm}$ and $4.72-8.8 \mathrm{~mm}$ for radially- and tangentially-oriented sources, respectively (Anwander et al. 2002; Güllmar et al. 2010), with one study reporting a maximum error of $26.3 \mathrm{~mm}$ (Hallez et al. 2005). Additionally, disregarding anisotropy had a large influence on the induced electric fields from TMS (De Lucia et al. 2007), whilst WM anisotropy influenced the electrical potential distribution following application of deep brain stimulation (Butson et al. 2007; McIntyre et al. 2004). Uncertainty in WM conductivity also had a significantly large effect on tDCS stimulation amplitudes and current density estimations, which were especially pronounced in the auditory cortex, implying orientation to be a determining factor in tDCS applications (Schmidt et al. 2015).

Isotropic WM conductivities however were found to vary dependent on method, measurement condition, pathology and age, additionally diverging with temperature and 
frequency. As previously observed, MREIT produced elevated WM conductivity values with larger variation between values compared to other methods, supporting the uncertainty and apparently low reliability of MREIT for estimating conductivity. Furthermore, DTI values produced similar, largely varying results. As previously discussed by Rullmann and colleagues (2009), elevated conductivity values from DTI may be a result of an overestimated scaling factor from the diffusion tensor to the conductivity tensor, proposed by the authors. In line with this, Akhtari and colleagues (2006) failed to verify this relationship and instead revealed an inverse linear relationship with a scaling factor of -0.367 , and considerable variability between values. For this reason, Güllmar et al. (2010) compensated for isotropic variation by normalising the conductivity tensors (by calculating the anisotropy ratio between eigenvalues) in one model and using fixed artificial anisotropy ratios to preserve diffusion tensor orientation, in another model (volume constraint model). By comparing both models with Tuch's, they revealed that employing the latter significantly affected MEG and EEG forward computations differently by changing the mean scalar representation of isotropic tensors. This study, alongside previous investigations, emphasised the importance of modelling anisotropy, but has also insinuated further, more detailed research should explore the linear relationship between the diffusion and electrical conductivity tensors.

Additionally, differences were revealed between perpendicularly- and parallelly-oriented WM conductivities that are due to the results being obtained from different papers. Of note, the minimum WM_par conductivity value $(0.0543 \mathrm{~S} / \mathrm{m})$ is less than the minimum WM-perp value $(0.0620 \mathrm{~S} / \mathrm{m})$, with only small differences reported between the means. These results are not indicative of WM conductivity themselves, but instead highlight the variation in values between studies. Moreover, this may reflect the differences between the methods employed for approximating the conductivity tensor from the diffusion tensor, due to the fact that WM is more anisotropic than these results indicate. A recent paper (Wu et al. 2018) reviewed the current anisotropic conductivity models of WM based on DTI. The linear relation model (i.e. Tuch's model) was discussed as not directly considering the impact of geometrical brain tissue structure, whilst the Wang-constraint (Wang et al. 2008) and volume-constraint models, both of which assume diffusion and conductivity tensors share the same eigenvalues (similar to Tuch's model), ignore brain tissue heterogeneity but can relate anisotropy and physiological structure. The equilibrium model (Sekino et al. 2005), which decomposes extracellular and intracellular diffusion, can be less accurate as extracellular diffusion may result difficult to quantify (Jones et al. 2018). Wu et al. (2018) determined that obtaining the conversion coefficient between the anisotropic conductivity tensor and the diffusion tensor eigenvalues to be of most importance. Further, they concluded the optimum model to be the electrochemical model, which calculates the conversion coefficient according to the concentration of charged particles in interstitial fluid and has the added benefit of being able to calculate a conversion coefficient for GM and avoids having to consider the effect WM structure has on water molecules and electrical charges. They noted however, that the models are not contradictory, but instead complement each other to inform the relationship between conductivity and diffusion tensors. This emphasises the need for more research to elucidate the prime conversion coefficient before robust conclusions can be made. Exploration and evaluation of different diffusion-to-conductivity methods are beyond the scope of the current meta-analysis, however, considering the variable results in combination with $\mathrm{Wu}$ et al. (2018) analysis, caution should be taken when applying conversion algorithms between conductivity and diffusion tensors.

Furthermore, as formerly discussed, measurements obtained in vitro or ex vivo, as well as at room temperature, are likely to differ from in vivo results due to the non-physiological conditions. It is therefore recommended investigations should be completed at body temperature, in vivo and at frequencies in line with resonant frequencies of the brain $(<100 \mathrm{~Hz})$. Subsequently, a significant correlation between WM conductivity and applied frequency may have only be revealed due to values obtained at $500 \mathrm{~Hz}$. A large pool of conductivity values were obtained at $500 \mathrm{~Hz}$, whilst the remainder of values were measured at frequencies $<150 \mathrm{~Hz}$, hence, the higher conductivity values at a considerably higher frequency than all other results may have skewed the data to reveal a positive relationship, which may not otherwise be there. Additional conductivity values measured with frequencies between 150 and $500 \mathrm{~Hz}$ are needed to further elucidate the presence or not of a significant relationship.

WM conductivity values were additionally revealed to vary with pathological condition, however insufficient results were available to extract clear conclusions. Such variation with pathology is nevertheless, expected, for example intracranial pathology from epilepsy patients potentially alters cytoarchitecture of affected and non-affected areas, such as cortical neuronal disorganisation and surplus WM cells (Mathern et al. 1999). Akhtari and colleagues (2006) suggested pathological changes in myelin and diseased-active cells may disrupt cell geometry organisation, which, when coupled with histological demyelination and cell population alterations, may increase proton diffusion as it is no longer constrained by myelin walls or tight organisation. Interestingly, a significant relationship between fluctuations in DTI eigenvalues and histological alterations in temporal lobe epilepsy has been found (Kimiwada et al. 2006). Furthermore, extensive research has revealed marked differences in 
WM structure in numerous diseases and pathologies, such as Multiple Sclerosis, Alzheimer's Disease, Schizophrenia and Parkinson's Disease (Bozzali et al. 2002; Burton et al. 2006; Kubicki et al. 2005; Kutzelnigg et al. 2005). Conductivity and anisotropy surrounding diseased tissues is subsequently likely to affect the generated electrical and magnetic fields from a source, compared to such distributions in healthy individuals (Park et al. 2002; Youn et al. 2003).

Although no significant correlation was revealed, WM conductivity as a function of age was revealed to contribute to the meta-regression model. This observation is unsurprising considering the well-researched nature of WM development with age, suggesting a decline in WM integrity, WM volume, myelination, diffusivity, etc. (Gunning-Dixon et al. 2009; Guttmann et al. 1998; Salat et al. 1999; Schmithorst et al. 2002). The degeneration of WM with age allows for higher CSF and liquid concentrations within the WM, therefore increasing conductivity of the tissue. Considering the large variation of WM with methodology, pathology and participant demographics, assuming conductivity of WM from the literature is clearly insufficient for accurate head conductivity profiles.

Combining the current results for WM and GM conductivity values, a clear discrepancy exists between both tissues, indicating the heterogeneity of the brain. Assuming the brain to have homogeneous and isotropic conductivity, as in research considering the brain as a whole, is therefore insufficient for accurate conductivity profiles. Assuming the brain as a homogeneous conductor consequently results in considerable EEG and MEG source localisation errors (Acar et al. 2016; Awada et al. 1998; Cohen and Cuffin 1983b).

\section{Brain-to-Skull Conductivity Ratio}

The ratio between brain and skull conductivity was significantly different for epilepsy compared to healthy participants, however, all BSCR values with epileptic pathology were obtained from paediatric samples. In accordance with this observation, BSCR was revealed to increase with age, suggesting paediatric samples, and hence epilepsy in the current review, to have lower conductivity ratios. In support of previous literature examining the influence of age on skull conductivity, such a relationship is expected, considering increased conductivity and decreased thickness of paediatric skulls. In contrast, paediatric brain tissue contains relatively higher water content and lower myelin deposition than adults (Knickmeyer et al. 2008; Peterson et al. 2003), indicating higher conductivity. Together, higher conductivity of both the skull and the brain in paediatric samples would suggest the brain-to-skull ratio to remain relatively stable throughout age. However, this would require an equal rate of decline for both tissues, which is unlikely the case. The role of skull conductivity for BSCR calculations was elucidated by (Goncalves et al. 2003a, b, c), who concluded their BSCR discrepancies to be a consequence of skull, as opposed to brain, conductivity variation. Their results indicated brain conductivity to be of less importance when calculating the brain-to-skull conductivity ratio, rendering decline in brain conductivity, whether equal or not to skull conductivity decline, irrelevant.

In contrast, assuming isotropic and homogeneous properties of the skull may contribute to variations in brain-to-skull estimations. For example, current injection at different locations may result in differing impedance distribution within the skull, hence altering BSCR estimations when isotropic compared to anisotropic skull models are used (van den Broek et al. 1998). However, it is noted that more recent papers acknowledge the importance of segmenting the skull layers, hence the use of skull anisotropy to optimise estimates of layered skull conductivity may not be required. Additionally, the heterogeneity in the skull will evidently introduce conductivity variation when homogeneous models are used, hence contributing to variation in BSCR values. These observations similarly apply for brain homogeneity and anisotropy, which would consequently affect BSCR estimations and contribute to variation. Considering BSCR estimations are clearly dependent on accurate conductivity of the skull and brain, which are subject to large variability, it is suggested personalised models of whole-head conductivity are essential to accurately determine the brain-to-skull conductivity ratio.

\section{Conclusion}

The current meta-analysis systematically investigated variation in reported human head electrical conductivity values for 17 different tissue types to evidence deviation within and between tissues, determine any influential factors and evaluate the impact on E/MEG source reconstruction. Adhering to the hypothesis, conductivity was revealed to significantly vary throughout the literature, specifically for the scalp, different layers of the skull, the whole-brain, grey matter, white matter and the brain-to-skull conductivity ratio. To decrease variation and increase stability of conductivity estimates, values should be obtained at body temperature, at frequencies $<100 \mathrm{~Hz}$ and in natural, in vivo conditions. Conductivity was significantly discovered to vary dependent on participant pathology, suggesting separate values should be acquired for different pathologies. However, further research was suggested to enhance the understanding of pathological effects on the electrical conductivity field. Additionally, electrical conductivity significantly correlated with participant's age for the skull, WM and BSCR. Previous literature was presented indicating large EEG and MEG source localisation errors occurring as a result of inaccurate conductivity values, particularly when neglecting anisotropy and heterogeneity of 
the head. The impact of incorrect conductivity on E/MEG forward and inverse solutions, coupled with the high variability of conductivity for each tissue type, suggests that assuming conductivity from the literature is insufficient. In conclusion, to optimise source reconstruction in EEG and MEG, and reduce localisation errors, personalised models of head electrical conductivity should be obtained for each individual.

Acknowledgements This work was supported by the Knowledge Economy Skills PhD Scholarship. Knowledge Economy Skills Scholarships (KESS) is a pan-Wales higher level skills initiative led by Bangor University on behalf of the HE sector in Wales. It is part funded by the Welsh Government's European Social Fund (ESF) convergence programme for West Wales and the Valleys.

\section{Compliance with Ethical Standards}

Conflict of interest The authors declare that they have no conflict of interest.

Open Access This article is distributed under the terms of the Creative Commons Attribution 4.0 International License (http://creativeco mmons.org/licenses/by/4.0/), which permits unrestricted use, distribution, and reproduction in any medium, provided you give appropriate credit to the original author(s) and the source, provide a link to the Creative Commons license, and indicate if changes were made.

\section{Appendix 1: Prisma Flow Diagram}

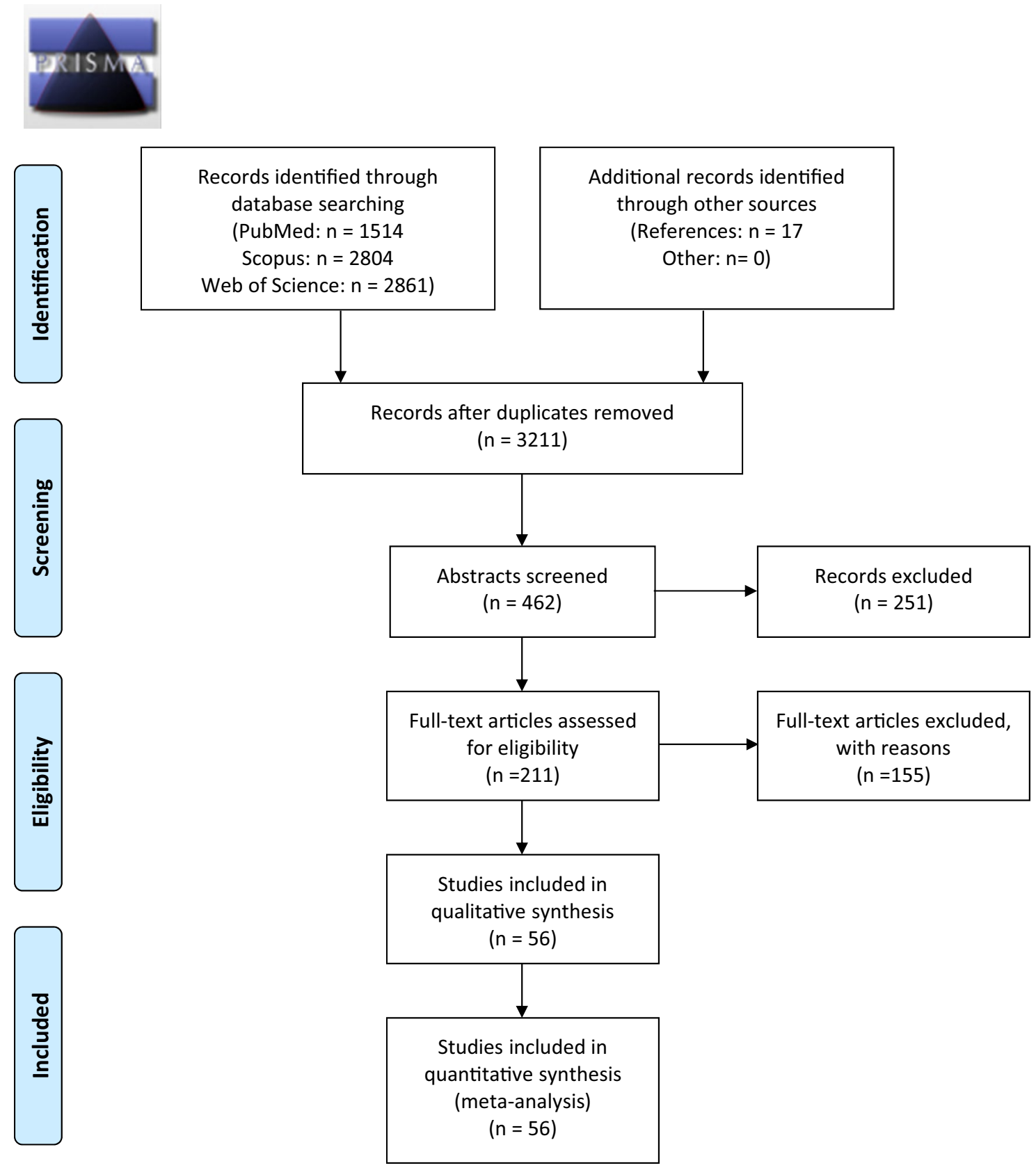


From Moher et al (2009).

For more information, visit www.prisma-statement.org.

\section{Appendix 2: Keywords for Literature Search}

The inclusion criteria considered Articles, Books, Book Chapters, Corrections, Data Paper, Early Access or Reprints presented or translatable to English. The keyword search was conducted for titles only. Keywords included any variation and combination of "conductivity" (i.e. resistance, impedance, dielectric, electric/current field, electric properties) AND "head tissue" (i.e. head, brain, scalp, skull, cerebral, CSF, dura, white matter, grey matter, brain-skull, brainscalp, BSCR, lesion). To reduce the amount of retrieved papers, those including unrelated keywords in their titles (i.e. insulin, diabetes, drug, DNA, blight, ship, sea, flower, Kawasaki, train) and non-human animals (i.e. rat, pig sheep, cow, swine, mice, mouse) were excluded from the keyword search.

\section{Appendix 3: Quality Assessment Protocol for All Studies}

Item 1: Were participants appropriately recruited and described?

Pre-specified protocol

Yes-1

No-0

Unclear- 0.5
Pre-specified protocol

Yes-1

No-0

Unclear-0.5

Yes-1

No-0 pathology were excluded in order to explore how variations affect conductivity values. However, both should be considered when analysing results to accurately determine their impact. Therefore, an accurate description of participants should be provided in order to appropriately group them for analysis. In the case no information is provided, it should be made clear participants are assumed to be healthy adults.

At minimum, participant pathology was provided. Age is further included in the current metaanalysis if available.

No information on participant pathology or demographics were provided, or sufficient to assume participants were healthy adults.

When no detailed description was available, it is still sufficient to assume participants were healthy adults. replicated?

Pre-specified protocol

Pre-specified protocol

Item 2: Does the measurement method appropriately determine the desired value?

The study measures what they set out to measure (i.e. conductivity/ resistivity) and reports the values appropriately.

Results were reported in accordance to the study aims and methodology.

Reported values were inconsistent with the study aims and what they claimed to report.

It is unclear whether or not reported results were in line with the studies aim, therefore an average of 0.5 for this item is assigned.

Item 3: Is the methodology accurately described such that it can be

Employed methodology should be sufficiently described in order to accurately assess variation in conductivity values due to methodological differences. Enough information should therefore, at minimum, be provided to assign methodology. In order to further validate the chosen method, a sufficient description should allow replication of the method.

Sufficient information was provided to assign a methodology and replicate their chosen method.

No information was provided, and the review is unable to appropriately assign chosen methodology.

Unclear $0.5 \quad$ It is unclear whether enough information is provided for replication, but methodology can be assigned appropriately.

Item 4: Is the chosen methodology justified?

As there are many different methods measuring conductivity, it would be expected each study would justify their chosen methodology, potentially based on the previous literature.

Yes-1 Justification for the chosen method was provided.

No-0 No justification for the employed methodology was provided.

Unclear-0.5 It is unclear whether or not justification was provided, therefore an average of 0.5 for this item is assigned.

Item 5: Was the measurement protocol verified for accuracy? 
Pre-specified protocol

No-0

Unclear- 0.5
Tests measuring conductivity do not come without errors, in order to improve validity of the method, measurement errors should be provided to further verify their results. This may be through simulation or phantom experiments, or as standard deviations within each participant.

The error associated with the chosen methodology was reportedfrom simulation, phantom or participant data.

No protocol errors were reported.

It is unclear whether errors were reported or not-i.e. unclear whether reported errors were due to simulation, phantom or participant data errors.

Item 6: Did the measurement and verification method remain unchanged throughout the study?

Pre-specified protocol

Methodology should remain constant throughout the measurement process, with any deviations described and justified.

Yes-1

All measurements were obtained using the same methodology.

No-0 Methodology did not remain constant.

Unclear- 0.5

It is unclear whether or not the methodology was consistent for each measurement, therefore an average of 0.5 for this item is assigned.

Item 7: Were the measurement errors within an acceptable range? Higher weight?

Pre-specified protocol

Yes-1

No-0

Unclear- 0.5
Errors should be of an acceptable value otherwise use of the chosen method is not justified. Where errors are considerably large, a discussion should be made as to why and ramifications implemented.

Errors were relatively low and within an acceptable range.

Errors were considerably large, and no justifications/ramifications were made.

No clear error values were provided, or justification/ ramification are absent/arbitrary, therefore an average of 0.5 for this item is assigned.

\section{Direct Measurement}

Item 8: Were measurements obtained immediately after tissue was excised? If no, how much time elapsed between excision and test? Pre-specified protocol

For conductivity values that reflect the most natural circumstances, measurements should be made immediately after tissue has been excised, or immediately after death. Time-elapsed from excision and measurements may affect the relative conductivity of the tissue.

Yes-1

No time elapsed from excision and test.

$<24$ h- 0.8

$1-7$ days -0.6

1-8 weeks- 0.4

$>2$ months -0.2

Time elapsed between excision and test within the range described and an item score was assigned accordingly.

Unclear- 0.5

It was unclear how much time elapsed, therefore an average of 0.5 for this item is assigned.

Item 9: Were excised tissue samples kept in $100 \%$ saline? If no, what liquid (and concentration) were tissue samples kept in?

Pre-specified protocol

To ensure conditions are kept as natural as possible, excised tissue should be contained within $100 \%$ saline, where conductivity is $1 \mathrm{~S} / \mathrm{m}$.

Yes-1

Excised tissue samples were contained in $100 \%$ saline of $1 \mathrm{~S} / \mathrm{m}$ conductivity.

No:

$>90 \%$ saline- 0.8

50-90\% saline- 0.6

$<50 \%$ saline- 0.4

Dry- 0.2

Unclear-0.5

Tissue samples were kept in varying concentrations of saline and quality scores were assigned accordingly.

It is unclear what concentration of saline tissues were kept in, hence they were assigned an average score of 0.5

Item 10: Was the tissue kept at body temperature $\left(\sim 37^{\circ} \mathrm{C}\right)$ ?

Pre-specified protocol

Yes-1

No-0

Unclear- 0.5
For conductivity values that reflect the most natural circumstances, excised tissue should be maintained at body temperature.

Tissue kept at approximately body temperature $\left(34-39^{\circ} \mathrm{C}\right)$

Tissue not at body temperature.

The temperature of the tissue during conductivity measurements was unclear or ambiguous, therefore an average of 0.5 for this item was assigned. 


\section{Model-Dependent Measurements}

Item 11: Is an individualised head model considered for each participant?

Pre-specified protocol

Yes, individualistic-1

No, realistic- 0.75

No, spherical- 0.25

Unclear- 0.5

Item 12: Has the head been maximally segmented into appropriate layers depending on the tissue type being measured?

Pre-specified protocol

12a: scalp

Yes: muscle, fat skin- 1

Yes: 2 layers- 0.5

No-0

N/A

12b: skull

Yes: four layers-1

Yes: three layers-0.66

Yes: two layers- 0.33

No: one layer- 0

N/A

12c: brain

Yes: $\mathrm{GM}+\mathrm{WM}-1$

No: homogenous- 0.5

3 tissue layers of muscle, fat, skin (i.e. muscle + fat) enous layer. conductivity, item 12a is not ment score.

The skull was segmented into spongiform, inner and outer compact bone with sutures.

Skull considered tri-layered; spongiform, inner and outer and compact bone. homogenous head layer. conductivity, item $12 \mathrm{~b}$ is not ment score. grey and white matter.

The brain was considered as a
Head shape and tissue thickness varies considerably between individuals; therefore, head models should be personalised for each individual - i.e. from MRI/DTI data. If not, realistic head models should be employed as opposed to a spherical model, as the head is not a simplistic sphere.

Individualistic head models were considered.

A realistic head model was employed.

A spherical head model was employed.

The head model used was unclear, hence an average quality score of 0.5 is given.
No: scalp $=$ brain -0

The scalp was segmented into the

The scalp is considered as 2 layers

Scalp is considered one homog-

If the study did not measure scalp included in the Quality Assesscompact bone without sutures.

Skull segmented into spongiform

The skull was considered as one

If the study did not measure scalp included in the Quality Assess-

The brain was compartmented into homogenous tissue.
N/A

12d: WM anisotropy

Yes: anisotropic-1

No- isotropic-0

N/A

Item 13: Were no assumptions made for the conductivity value of any tissue type?

Pre-specified protocol

Yes-1

No-0

The brain was assumed to have the same conductivity as the scalp.

If the study did not measure brain conductivity, item 12c is not included in the Quality Assessment score.

WM was modelled as anisotropic

WM was modelled as isotropic, or this was not mentioned in the study, therefore WM was assumed to be modelled as isotropic

If the study did not measure WM conductivity, item $12 \mathrm{~d}$ is not included in the Quality Assessment score. reported tissue types were empirically measured rather than assumed from prior literature (i.e. CSF is often assumed to model the remaining tissues).

No assumptions were made

Conductivity was assumed for one or more tissue types.

\section{Model-Independent Measurements}

Item 14: Was the magnetic resonance resolution high?

Pre-specified protocol

High resolution magnetic resonance imaging data with small voxel sizes should be acquired in order to most accurately segment head tissue and improve spatial resolution.

Yes: $<1 \mathrm{~mm}-1$

No: $1-2$ mm- 0.8

MR resolution is $1 \mathrm{~mm}^{3}$ or less.

No: $2-3 \mathrm{~mm}-0.6$

Resolution between 1 and $2 \mathrm{~mm}^{3}$.

No: 3-4 mm-0.4

No: $>4$ mm- 0.2

Unclear-0.5

Resolution above $4 \mathrm{~mm}^{3}$.

The resolution used was unclear or unprovided, hence an average quality score of 0.5 is given.

N/A

If the study did not employ a magnetic resonance method, item 14 is not included in the Quality Assessment score.

\section{Example 1 \& 2: Direct Measurements}

Baumann et al. (1997) 


\begin{tabular}{|c|c|c|c|c|c|c|c|c|c|c|c|c|c|c|c|c|c|}
\hline Item & 1 & 2 & 3 & 4 & 5 & 6 & 7 & 8 & 9 & 10 & 11 & $12 a$ & $12 b$ & $12 \mathrm{c}$ & $12 d$ & 13 & 14 \\
\hline Score & 1 & 1 & 1 & 1 & 0 & 1 & 0.5 & 0.4 & 0.5 & 1 & N/A & N/A & N/A & N/A & N/A & N/A & N/A \\
\hline Total & & 7.4 & & & & & & & & & & & & & & & \\
\hline QAS & & 0.7 & $4(\mathrm{Sc}$ & re & tal & ivid & $d$ by $n$ & nber $\mathrm{c}$ & celeva & item & in this & e 10) & & & & & \\
\hline
\end{tabular}

Akhtari et al. (2000)

\begin{tabular}{|c|c|c|c|c|c|c|c|c|c|c|c|c|c|c|c|c|c|}
\hline Item & 1 & 2 & 3 & 4 & 5 & 6 & 7 & 8 & 9 & 10 & 11 & $12 \mathrm{a}$ & $12 b$ & $12 \mathrm{c}$ & $12 \mathrm{~d}$ & 13 & 14 \\
\hline Score & 1 & 1 & 1 & 1 & 1 & 1 & 1 & 0.2 & 0.8 & 1 & N/A & N/A & N/A & N/A & N/A & N/A & N/A \\
\hline Total & & 9 & & & & & & & & & & & & & & & \\
\hline QAS & & & (Scc & to & al di & idec & by $n$ & Imber & releva & item & in this & e 10) & & & & & \\
\hline
\end{tabular}

\section{Example 3 and 4: Model Dependent Measurements}

Dannhauer et al. (2011)—E/MEG

\begin{tabular}{llllllllllllllllll}
\hline Item & 1 & 2 & 3 & 4 & 5 & 6 & 7 & 8 & 9 & 10 & 11 & $12 \mathrm{a}$ & $12 \mathrm{~b}$ & $12 \mathrm{c}$ & $12 \mathrm{~d}$ & 13 & 14 \\
\hline Score & 1 & 1 & 1 & 1 & 0 & 1 & 0.5 & N/A & N/A & N/A & 1 & 0 & 0.66 & 1 & N/A & 0 & N/A \\
Total & & 8.16 & & & & & & & & & & & \\
QAS & & & & & & & & & & \\
\hline
\end{tabular}

Fernández-Corazza et al. (2017) EIT

\begin{tabular}{llllllllllllllllll}
\hline Item & 1 & 2 & 3 & 4 & 5 & 6 & 7 & 8 & 9 & 10 & 11 & $12 \mathrm{a}$ & $12 \mathrm{~b}$ & $12 \mathrm{c}$ & $12 \mathrm{~d}$ & 13 & 14 \\
\hline Score & 1 & 1 & 1 & 1 & 1 & 1 & 1 & N/A & N/A & N/A & 1 & 0 & 0.33 & 1 & N/A & 0 & N/A \\
Total & & 9.33 & & & & & & & & & & & & & & \\
QAS & & & & & & & & & & \\
\hline
\end{tabular}

\section{Example 5 and 6: Model Independent \\ Measurements}

Rullmann et al. (2009)—DTI

\begin{tabular}{|c|c|c|c|c|c|c|c|c|c|c|c|c|c|c|c|c|c|}
\hline Item & 1 & 2 & 3 & 4 & 5 & 6 & 7 & 8 & 9 & 10 & 11 & $12 a$ & $12 b$ & $12 \mathrm{c}$ & $12 d$ & 13 & 14 \\
\hline Score & 1 & 1 & 1 & 1 & 1 & 1 & 1 & N/A & N/A & N/A & N/A & N/A & N/A & N/A & N/A & N/A & 1 \\
\hline Total & & 8 & & & & & & & & & & & & & & & \\
\hline QAS & & & cor & tot & $\operatorname{div}$ & ded & $\mathrm{y} n$ & nber of & levant & $\mathrm{ns}$, in $\mathrm{t}$ & case 8) & & & & & & \\
\hline
\end{tabular}

Voigt et al. (2011)—MREIT

\begin{tabular}{|c|c|c|c|c|c|c|c|c|c|c|c|c|c|c|c|c|c|}
\hline Item & 1 & 2 & 3 & 4 & 5 & 6 & 7 & 8 & 9 & 10 & 11 & $12 \mathrm{a}$ & $12 \mathrm{~b}$ & $12 \mathrm{c}$ & $12 \mathrm{~d}$ & 13 & 14 \\
\hline Score & 1 & 1 & 1 & 1 & 1 & 1 & 0.5 & N/A & N/A & N/A & N/A & N/A & N/A & N/A & N/A & N/A & 0.8 \\
\hline Total & & 7.3 & & & & & & & & & & & & & & & \\
\hline QAS & & 0.9 & 125 & $\mathrm{Scc}$ & tot & $\mathrm{ll} \mathrm{di}$ & ided b & numbe & f relev & items, & this ca & & & & & & \\
\hline
\end{tabular}




\section{References}

Acar, Z. A., Ortiz-Mantilla, S., Benasich, A., \& Makeig, S. (2016, Aug 16-20). High-resolution EEG source imaging of one-yearold children. Paper presented at the 38th Annual International Conference of the IEEE-Engineering-in-Medicine-and-BiologySociety (EMBC), Orlando

Akhtari M, Bryant HC, Mamelak AN, Heller L, Shih JJ, Mandelkern M, Ranken DM, Best ED, Sutherling WW (2000) Conductivities of three-layer human skull. Brain Topogr 13(1):29-42. https:// doi.org/10.1023/a:1007882102297

Akhtari M, Bryant HC, Marnelak AN, Flynn ER, Heller L, Shih JJ, Mandelkern M, Ranken DM, Best ED, DiMauro MA (2002) Conductivities of three-layer live human skull. Brain Topogr 14(3):151-167. https://doi.org/10.1023/a:1014590923185

Akhtari M, Bryant H, Emin D, Merrifield W, Mamelak A, Flynn E, Shih JJ, Mandelkern M, Matlachov A, Ranken DM, Best ED (2003) A model for frequency dependence of conductivities of the live human skull. Brain Topogr 16(1):39-55

Akhtari M, Salamon N, Duncan R, Fried I, Mathern GW (2006) Electrical conductivities of the freshly excised cerebral cortex in epilepsy surgery patients; Correlation with pathology, seizure duration, and diffusion tensor imaging. Brain Topogr 18(4):281-290. https://doi.org/10.1007/s10548-006-0006-x

Akhtari M, Mandelkern M, Bui D, Salamon N, Vinters HV, Mathern GW (2010) Variable Anisotropic Brain Electrical Conductivities in Epileptogenic Foci. Brain Topogr 23(3):292-300. https://doi. org/10.1007/s10548-010-0144-z

Akhtari M, Emin D, Ellingson BM, Woodworth D, Frew A, Mathern GW (2016) Measuring the local electrical conductivity of human brain tissue. J Appl Phys 119(6):064701. https://doi. org/10.1063/1.4941556

Antonakakis M, Oostenveld R, Wellmer J, Möddel G, Haueisen J, Rampp S, Wolters C (2018) Age-related skull conductivity estimated by a calibration procedure using combined somatosensory evoked potentials and fields on realistic head models. In: Paper presented at 50 years of MEG, ISACM/EMEGS conference, Poros, Greece, April 2018

Anwander A, Wolters CH, Dümpelmann M, Knösche T (2002) Influence of realistic skull and white matter anisotropy on the inverse problem in EEG/MEG-source localization. In: Paper presented at the Proceedings of The 13th International Conference on Biomagnetism

Arumugam ET, Sergei P, Nick R, Dennis PL, Tucker P, Don (2017) Invivo estimation of the scalp and skull conductivity using bEIT for non-invasive neuroimaging and stimulation. In: Brain stimulation and imaging meeting, Vancouver

Awada KA, Jackson DR, Baumann SB, Williams JT, Wilton DR, Fink PW, Prasky BR (1998) Effect of conductivity uncertainties and modeling errors on EEG source localization using a 2-D model. IEEE Trans Biomed Eng 45(9):1135-1145

Aydin Ü, Vorwerk J, Kupper P, Heers M, Kugel H, Galka A, Hamid L, Wellmer J, Kellinghaus C, Rampp S, Wolters CH (2014) Combining EEG and MEG for the reconstruction of epileptic activity using a calibrated realistic volume conductor model. PLoS ONE 9(3):e93154. https://doi.org/10.1371/journal.pone.0093154

Aydin Ü, Rampp S, Wollbrink A, Kugel H, Cho J-H, Knösche TR, Grova C, Wellmer J, Wolters C (2017) Zoomed MRI guided by combined EEG/MEG source analysis: a multimodal approach for optimizing presurgical epilepsy work-up and its application in a multi-focal epilepsy patient case study. Brain Topogr 30(4):417-433

Barber DC, Brown BH (1984) Applied potential tomography. J Phys E 19(9):220-223
Baumann SB, Wozny DR, Kelly SK, Meno FM (1997) The electrical conductivity of human cerebrospinal fluid at body temperature. IEEE Trans Biomed Eng 44(3):220-223. https://doi. org/10.1109/10.554770

Baysal U, Haueisen J (2004a) Use of a priori information in estimating tissue resistivities-application to human data in vivo. Physiol Meas 25(3):737-748. https://doi. org/10.1088/0967-3334/25/3/013

Baysal U, Haueisen J (2004b) Use of a priori information in estimating tissue resistivities - application to human data in vivo. Phys Meas 25:737-748

Beltrachini L (2019a) Sensitivity of the projected subtraction approach to mesh degeneracies and its impact on the forward problem in EEG. IEEE Trans Biomed Eng 66(1):273-282

Beltrachini L (2019b) A finite element solution of the forward problem in EEG for multipolar sources. IEEE Trans Neural Syst Rehabil Eng 27(3):368-377. https://doi.org/10.1109/tnsre.2018.2886638

Berényi A, Belluscio M, Mao D, Buzsáki G (2012) Closed-loop control of epilepsy by transcranial electrical stimulation. Science 337(6095):735-737

Birot G, Spinelli L, Vulliémoz S, Mégevand P, Brunet D, Seeck M, Michel CM (2014) Head model and electrical source imaging: a study of 38 epileptic patients. NeuroImage Clin 5:77-83

Bodenstein M, David M, Markstaller K (2009) Principles of electrical impedance tomography and its clinical application. Crit Care Med 37(2):713-724. https://doi.org/10.1097/CCM.0b013e3181 $958 \mathrm{~d} 2 \mathrm{f}$

Borenstein M, Hedges LV, Higgins JP, Rothstein HR (2011) Introduction to meta-analysis. Wiley, Chichester

Bozzali M, Falini A, Franceschi M, Cercignani M, Zuffi M, Scotti G, Comi G, Filippi M (2002) White matter damage in Alzheimer's disease assessed in vivo using diffusion tensor magnetic resonance imaging. J Neurol Neurosurg Psychiatry 72(6):742-746

Brodbeck V, Spinelli L, Lascano AM, Wissmeier M, Vargas M-I, Vulliemoz S, Pollo C, Schaller K, Michel CM, Seeck M (2011) Electroencephalographic source imaging: a prospective study of 152 operated epileptic patients. Brain 134(10):2887-2897

Brunoni AR, Fregni F, Priori A, Ferrucci R, Boggio PS (2013) Transcranial direct current stimulation: challenges, opportunities, and impact on psychiatry and neurorehabilitation. Front Psychiatry $4: 19$

Burger H, Van Dongen R (1961) Specific electric resistance of body tissues. Phys Med Biol 5(4):431

Burger HC, van Milaan JB (1943) Measurements of the specific resistance of the human body to direct current. Acta Med Scand 114(6):584-607

Burton EJ, McKeith IG, Burn DJ, Firbank MJ, O’Brien JT (2006) Progression of white matter hyperintensities in Alzheimer disease, dementia with lewy bodies, and Parkinson disease dementia: a comparison with normal aging. Am J Geriatr Psychiatry 14(10):842-849

Butson CR, Cooper SE, Henderson JM, McIntyre CC (2007) Patientspecific analysis of the volume of tissue activated during deep brain stimulation. Neuroimage 34(2):661-670

Bystron I, Blakemore C, Rakic P (2008) Development of the human cerebral cortex: boulder Committee revisited. Nat Rev Neurosci $9(2): 110$

Chauhan M, Indahlastari A, Kasinadhuni AK, Schär M, Mareci TH, Sadleir RJ (2018) Low-frequency conductivity tensor imaging of the human head in vivo using DT-MREIT: first study. IEEE Trans Med Imaging 37(4):966-976

Chauveau N, Franceries X, Doyon B, Rigaud B, Morucci JP, Celsis P (2004) Effects of skull thickness, anisotropy, and inhomogeneity on forward EEG/ERP computations using a spherical threedimensional resistor mesh model. Hum Brain Mapp 21(2):86-97 
Cho J-H, Vorwerk J, Wolters CH, Knösche TR (2015) Influence of the head model on EEG and MEG source connectivity analyses. Neuroimage 110:60-77

Christie A (1949) Prevalence and distribution of ossification centers in the newborn infant. Am J Dis Child 77(3):355-361

Clerc M, Adde G, Kybic J, Papadopoulo T, Badier J-M (2005) In vivo conductivity estimation with symmetric boundary elements. Int J Bioelectromagn 7:307-310

Cohen D, Cuffin BN (1983a) Demonstration of useful differences between magnetoencephalogram and electroencephalogram. Electroencephalogr Clin Neurophysiol 56(1):38-51. https://doi. org/10.1016/0013-4694(83)90005-6

Cohen D, Cuffin BN (1983b) Demonstration of useful differences between magnetoencephalogram and electroencephalogram. Electroencephalogr Clin Neurophysiol 56(1):38-51. https://doi. org/10.1016/0013-4694(83)90005-6

Compta Y, Ibarretxe-Bilbao N, Pereira JB, Junqué C, Bargalló N, Tolosa E, Valldeoriola F, Munoz E, Camara A, Buongiorno M, Marti MJ (2012) Grey matter volume correlates of cerebrospinal markers of Alzheimer-pathology in Parkinson's disease and related dementia. Parkinsonism Relat Disord 18(8):941-947

Corp I (2013) IBM SPSS statistics for Macintosh. Version 22.0. IBM Corp., Armonk

Dabek J, Kalogianni K, Rotgans E, van der Helm FCT, Kwakkel G, van Wegen EEH, Daffertshofer A, de Munck JC (2016) Determination of head conductivity frequency response in vivo with optimized EIT-EEG. Neuroimage 127:484-495. https://doi. org/10.1016/j.neuroimage.2015.11.023

Dannhauer M, Lanfer B, Wolters CH, Knosche TR (2011) Modeling of the human skull in EEG source analysis. Hum Brain Mapp 32(9):1383-1399. https://doi.org/10.1002/hbm.21114

De Lucia M, Parker GJM, Embleton K, Newton JM, Walsh V (2007) Diffusion tensor MRI-based estimation of the influence of brain tissue anisotropy on the effects of transcranial magnetic stimulation. Neuroimage 36(4):1159-1170. https://doi.org/10.1016/j. neuroimage.2007.03.062

Despotovic I, Cherian PJ, De Vos M, Hallez H, Deburchgraeve W, Govaert P, Lequin M, Visser GH, Swarte RM, Vansteenkiste E, Van Huffel S (2013) Relationship of EEG sources of neonatal seizures to acute perinatal brain lesions seen on MRI: a pilot study. Hum Brain Mapp 34(10):2402-2417

Dobbing J, Sands J (1973) Quantitative growth and development of human brain. Arch Dis Child 48(10):757-767

Douaud G, Smith S, Jenkinson M, Behrens T, Johansen-Berg H, Vickers J, James S, Votes N, Watkins K, Matthews PM, James A (2007) Anatomically related grey and white matter abnormalities in adolescent-onset schizophrenia. Brain 130(9):2375-2386

Erasmie U, Ringertz H (1976) Normal width of cranial sutures in the neonate and infant: an objective method of assessment. Acta Radiol Diagn 17(5A):565-572

Eriksen KJ (1990) In vivo human head regional conductivity estimation using a three-sphere model. Paper presented at the Engineering in Medicine and Biology Society, 1990. In: Proceedings of the twelfth annual international conference of the IEEE

Fabrizi L, Sparkes M, Horesh L, Abascal J, McEwan A, Bayford RH, Elwes R, Binnie CD, Holder DS (2006) Factors limiting the application of electrical impedance tomography for identification of regional conductivity changes using scalp electrodes during epileptic seizures in humans. Physiol Meas 27(5):S163-S174. https://doi.org/10.1088/0967-3334/27/5/s14

Faes TJC, van der Meij HA, de Munck JC, Heethaar RM (1999) The electric resistivity of human tissues $(100 \mathrm{~Hz}-10 \mathrm{MHz})$ : a metaanalysis of review studies. Physiol Meas 20(4):R1-R10. https:// doi.org/10.1088/0967-3334/20/4/201

Fernández-Corazza M, Turovets S, Luu P, Price N, Muravchik C, Tucker D (2017) Skull modeling effects in conductivity estimates using parametric electrical impedance tomography. IEEE Trans Biomed Eng 65(8):1785-1797

Fiederer LDJ, Vorwerk J, Lucka F, Dannhauer M, Yang S, Dümpelmann M, Schulze-Bonhage A, Aertsen A, Speck O, Wolters CH (2016) The role of blood vessels in high-resolution volume conductor head modeling of EEG. NeuroImage 128:193-208

Fishman RA (1992) Cerebrospinal fluid in diseases of the nervous system. Saunders, Philadelphia

Flemming L, Wang Y, Caprihan A, Eiselt M, Haueisen J, Okada Y (2005) Evaluation of the distortion of EEG signals caused by a hole in the skull mimicking the fontanel in the skull of human neonates. Clin Neurophysiol 116(5):1141-1152

Frantseva M, Cui J, Farzan F, Chinta LV, Velazquez JLP, Daskalakis ZJ (2014) Disrupted cortical conductivity in schizophrenia: tMS-EEG Study. Cereb Cortex 24(1):211-221. https://doi. org/10.1093/cercor/bhs304

Frisoni GB, Pievani M, Testa C, Sabattoli F, Bresciani L, Bonetti M, Beltramello A, Hayashi KM, Toga AW, Thompson PM (2007) The topography of grey matter involvement in early and late onset Alzheimer's disease. Brain 130(3):720-730

Gabriel C, Gabriel S, Corthout E (1996a) The dielectric properties of biological tissues: I. Literature survey. Phys Med Biol 41(11):2231

Gabriel C, Gabriel S, Corthout E (1996b) The dielectric properties of biological tissues: I. Literature survey. Phys Med Biol 41(11):2231

Gattellaro G, Minati L, Grisoli M, Mariani C, Carella F, Osio M, Ciceri E, Albanaese A, Bruzzone M (2009) White matter involvement in idiopathic Parkinson disease: a diffusion tensor imaging study. Am J Neuroradiol 30(6):1222-1226

Geddes AL, Baker LE (1967) The specific resistance of biological material - a compendium of data for the biomedical engineer and physiologist. Med Biol Eng 5(3):271-293

Gençer NG, Acar CE (2004a) Sensitivity of EEG and MEG measurements to tissue conductivity. Phys Med Biol 49(5):701

Gençer NG, Acar CE (2004b) Sensitivity of EEG and MEG measurements to tissue conductivity. Phys Med Biol 49:701

Geurts JJ, Barkhof F (2008) Grey matter pathology in multiple sclerosis. Lancet Neurol 7(9):841-851

Gibson A, Bayford R, Holder DS (2000) Two-dimensional finite element modelling of the neonatal head. Physiol Meas 21(1):45

Giorgio A, Santelli L, Tomassini V, Bosnell R, Smith S, De Stefano N, Johansen-Berg H (2010) Age-related changes in grey and white matter structure throughout adulthood. Neuroimage 51(3):943-951

Goncalves S, de Munck JC, Verbunt JPA, Heethaar RM, da Silva FHL (2003a) In vivo measurement of the brain and skull resistivities using an EIT-based method and the combined analysis of SEF/ SEP data. IEEE Trans Biomed Eng 50(9):1124-1128. https://doi. org/10.1109/tbme.2003.816072

Goncalves SI, de Munck JC, Verbunt JPA, Bijma F, Heethaar RM, da Silva FL (2003b) In vivo measurement of the brain and skull resistivities using an EIT-based method and realistic models for the head. IEEE Trans Biomed Eng 50(6):754-767. https://doi. org/10.1109/tbme.2003.812164

Goncalves SI, de Munck JC, Verbunt JPA, Bijma F, Heethaar RM, da Silva FL (2003c) In vivo measurement of the brain and skull resistivities using an EIT-based method and realistic models for the head Ieee Transactions on. Biomed Eng 50:754-767

Greimel E, Nehrkorn B, Schulte-Rüther M, Fink GR, Nickl-Jockschat T, Herpertz-Dahlmann B, Konrad K, Eickhoff SB (2013) Changes in grey matter development in autism spectrum disorder. Brain Struct Funct 218(4):929-942

Groeschel S, Vollmer B, King M, Connelly A (2010) Developmental changes in cerebral grey and white matter volume from infancy to adulthood. Int J Dev Neurosci 28(6):481-489 
Groppe DM, Bickel S, Keller CJ, Jain SK, Hwang ST, Harden C, Mehta AD (2013) Dominant frequencies of resting human brain activity as measured by the electrocorticogram. Neuroimage 79:223-233

Güllmar D, Haueisen J, Reichenbach JR (2010) Influence of anisotropic electrical conductivity in white matter tissue on the EEG/ MEG forward and inverse solution. A high-resolution whole head simulation study. Neuroimage 51(1):145-163. https://doi. org/10.1016/j.neuroimage.2010.02.014

Gunning-Dixon FM, Brickman AM, Cheng JC, Alexopoulos GS (2009) Aging of cerebral white matter: a review of MRI findings. Int J Geriatr Psychiatry 24(2):109-117

Gurler N, Ider YZ (2017) Gradient-based electrical conductivity imaging using MR phase. Magn Reson Med 77(1):137-150

Gutierrez D, Nehorai A, Muravchik CH (2004) Estimating brain conductivities and dipole source signals with EEG arrays. IEEE Trans Biomed Eng 51(12):2113-2122. https://doi.org/10.1109/ tbme.2004.836507

Guttmann CR, Jolesz FA, Kikinis R, Killiany RJ, Moss MB, Sandor T, Albert MS (1998) White matter changes with normal aging. Neurology 50(4):972-978

Haacke E, Petropoulos L, Nilges E, Wu D (1991) Extraction of conductivity and permittivity using magnetic resonance imaging. Phys Med Biol 36(6):723

Håkansson B, Brandt A, Carlsson P, Tjellström A (1994) Resonance frequencies of the human skull in vivo. J Acoustl Soc Am 95(3):1474-1481

Hallez H, Vanrumste B, Van Hese P, D’Asseler Y, Lemahieu I, Van de Walle R (2005) A finite difference method with reciprocity used to incorporate anisotropy in electroencephalogram dipole source localization. Phys Med Biol 50(16):3787-3806. https:// doi.org/10.1088/0031-9155/50/16/009

Hampe N, Herrmann M, Amthor T, Findeklee C, Doneva M, Katscher U (2018) Dictionary-based electric properties tomography. Magn Reson Med 81(1):342-349

Hansman CF (1966) Growth of interorbital distance and skull thickness as observed in roentgenographic measurements. Radiology 86(1):87-96

Hatipoglu HG, Ozcan HN, Hatipoglu US, Yuksel E (2008) Age, sex and body mass index in relation to calvarial diploe thickness and craniometric data on MRI. Forensic Sci Int 182(1-3):46-51

Haueisen J, Ramon C, Czapski P, Eiselt M (1995) On the influence of volume currents and extended sources on neuromagnetic fieldsa simulation study. Ann Biomed Eng 23(6):728-739. https://doi. org/10.1007/bf02584472

Haueisen J, Ramon C, Eiselt M, Brauer H, Nowak H (1997) Influence of tissue resistivities on neuromagnetic fields and electric potentials studied with a finite element model of the head. IEEE Trans Biomed Eng 44(8):727-735. https://doi.org/10.1109/10.605429

Haueisen J, Büttner A, Nowak H, Brauer H, Weiller C (1999) The influence of conductivity changes in boundary element compartments on the forward and inverse problem in electroencephalography and magnetoencephalography-Der Einfluß der Änderung der Schalenleitfähigkeit bei Randelementemodellen auf die Vorwärtsrechnung und das inverse problem in Elektroenzephalographie und Magnetoenzephalographie. Biomed Technik/Biomed Eng 44(6):150-157

Haueisen J, Tuch DS, Ramon C, Schimpf PH, Wedeen VJ, George JS, Belliveau JW (2002) The influence of brain tissue anisotropy on human EEG and MEG. Neuroimage 15(1):159-166. https://doi. org/10.1006/nimg.2001.0962

Henderson RP, Webster JG (1978) An impedance camera for spatially specific measurements of the thorax. IEEE Trans Biomed Eng 3(20):250-254

Hoekema R, Wieneke GH, Leijten FSS, van Veelen CWM, van Rijen PC, Huiskamp GJM, Ansems J, van Huffelen AC (2003) Measurement of the conductivity of skull, temporarily removed during epilepsy surgery. Brain Topogr 16(1):29-38. https://doi. org/10.1023/a:1025606415858

Huang Y, Liu AA, Lafon B, Friedman D, Dayan M, Wang XY, Bikson M, Doyle WK, Devinsky O, Parra LC (2017) Measurements and models of electric fields in the in vivo human brain during transcranial electric stimulation. Elife. https://doi.org/10.7554/ elife. 18834

Huhndorf M, Stehning C, Rohr A, Helle M, Katscher U, Jansen O (2013) Systematic brain tumor conductivity study with optimized EPT sequence and reconstruction algorithm. Paper presented at the Proc. ISMRM

Ishida H, Dodo Y (1990) Cranial thickness of modern and Neolithic populations in Japan. Hum Biol 62:389-401

Job DE, Whalley HC, Johnstone EC, Lawrie SM (2005) Grey matter changes over time in high risk subjects developing schizophrenia. Neuroimage 25(4):1023-1030

Johansen-Berg H, Behrens TE (2013) Diffusion MRI: from quantitative measurement to in vivo neuroanatomy. Academic Press, Amsterdam

Jones DK, Alexander DC, Bowtell R, Cercignani M, Dell'Acqua F, McHugh DJ, Tax CMW (2018) Microstructural imaging of the human brain with a 'super-scanner': 10 key advantages of ultrastrong gradients for diffusion MRI. NeuroImage 182:8-38

Kalu U, Sexton C, Loo C, Ebmeier K (2012) Transcranial direct current stimulation in the treatment of major depression: a meta-analysis. Psychol Med 42(9):1791-1800

Kim DH, Choi N, Gho SM, Shin J, Liu C (2014) Simultaneous imaging of in vivo conductivity and susceptibility. Magn Reson Med 71(3):1144-1150

Kimiwada T, Juhász C, Makki M, Muzik O, Chugani DC, Asano E, Chugani HT (2006) Hippocampal and thalamic diffusion abnormalities in children with temporal lobe epilepsy. Epilepsia 47(1):167-175

Knickmeyer RC, Gouttard S, Kang C, Evans D, Wilber K, Smith JK, Hamer RM, Lin W, Gerig G, Gilmore JH (2008) A structural MRI study of human brain development from birth to 2 years. J Neurosci 28(47):12176-12182

Koessler L, Colnat-Coulbois S, Cecchin T, Hofmanis J, Dmochowski JP, Norcia AM, Maillard LG (2017) In-vivo measurements of human brain tissue conductivity using focal electrical current injection through intracerebral multicontact electrodes. Hum Brain Mapp 38(2):974-986. https://doi.org/10.1002/hbm.23431

Kosterich JD, Foster KR, Pollack SR (1983) Dielectric permittivity and electrical conductivity of fluid saturated bone. IEEE Trans Biomed Eng 2:81-86

Kosterich JD, Foster KR, Pollack SR (1984) Dielectric properties of fluid-saturated bone- the effect of variation in conductivity of immersion fluid. IEEE Trans Biomed Eng 4:369-374

Kubicki M, Park H, Westin C-F, Nestor PG, Mulkern RV, Maier SE, Niznikiewicz M, Connor EE, Levitt JJ, Frumin M (2005) DTI and MTR abnormalities in schizophrenia: analysis of white matter integrity. Neuroimage 26(4):1109-1118

Kutzelnigg A, Lucchinetti CF, Stadelmann C, Brück W, Rauschka H, Bergmann M, Schmidbauer M, Parisi JE, Lassmann H (2005) Cortical demyelination and diffuse white matter injury in multiple sclerosis. Brain 128(11):2705-2712

Lai Y, van Drongelen W, Ding L, Hecox KE, Towle VL, Frim DM, He B (2005) Estimation of in vivo human brain-to-skull conductivity ratio from simultaneous extra- and intra-cranial electrical potential recordings. Clin Neurophysiol 116(2):456-465. https://doi. org/10.1016/j.clinph.2004.08.017

Lanfer B, Scherg M, Dannhauer M, Knösche TR, Burger M, Wolters CH (2012) Influences of skull segmentation inaccuracies on EEG source analysis. NeuroImage 62(1):418-431

Lau S, Güllmar D, Flemming L, Grayden DB, Cook MJ, Wolters CH, Haueisen J (2016) Skull defects in finite element head models 
for source reconstruction from magnetoencephalography signals. Front Neurosci 10:141

Law SK (1993) Thickness and resistivity variations over the upper surface of the human skull. Brain Topogr 6(2):99-109

Lee S-K, Bulumulla S, Wiesinger F, Sacolick L, Sun W, Hancu I (2015) Tissue electrical property mapping from zero echo-time magnetic resonance imaging. IEEE Trans Med Imaging 34(2):541-550

Lee J, Shin J, Kim DH (2016) MR-based conductivity imaging using multiple receiver coils. Magn Reson Med 76(2):530-539

Lew S, Sliva DD, Choe M-S, Grant PE, Okada Y, Wolters CH, Hämäläinen MS (2013) Effects of sutures and fontanels on MEG and EEG source analysis in a realistic infant head model. Neuroimage $76: 282-293$

Li Z, Park B-K, Liu W, Zhang J, Reed MP, Rupp JD, Hoff CN, Hu J (2015) A statistical skull geometry model for children 0-3 years old. PLoS ONE 10(5):e0127322

Liebetanz D, Klinker F, Hering D, Koch R, Nitsche MA, Potschka H, Loscher W, Paulus W, Tergau F (2006) Anticonvulsant effects of transcranial direct-current stimulation (tDCS) in the rat cortical ramp model of focal epilepsy. Epilepsia 47(7):1216-1224

Lynnerup N (2001) Cranial thickness in relation to age, sex and general body build in a Danish forensic sample. Forensic Sci Int 117(1-2):45-51

Lynnerup N, Astrup JG, Sejrsen B (2005) Thickness of the human cranial diploe in relation to age, sex and general body build. Head Face Med 1(1):13

Nurul AAL, Mahmood D, Mohd MK, Ibrahim S (2010) A study of frequency effects on conductivity measurements. RnD Seminar 2010: research and Development Seminar 2010, Malaysia

Mathern GW, Giza CC, Yudovin S, Vinters HV, Peacock WJ, Shewmon DA, Shields WD (1999) Postoperative seizure control and antiepileptic drug use in pediatric epilepsy surgery patients: the UCLA experience, 1986-1997. Epilepsia 40(12):1740-1749

McAlonan GM, Cheung V, Cheung C, Chua SE, Murphy DG, Suckling J, Tai KS, Yip LK, Leung P, Ho TP (2007) Mapping brain structure in attention deficit-hyperactivity disorder: a voxel-based MRI study of regional grey and white matter volume. Psychiatry Res 154(2):171-180

McGale E, Pye I, Stonier C, Hutchinson E, Aber G (1977) Studies of the inter-relationship between cerebrospinal fluid and plasma amino acid concentrations in normal individuals. J Neurochem 29(2):291-297

McIntyre CC, Mori S, Sherman DL, Thakor NV, Vitek JL (2004) Electric field and stimulating influence generated by deep brain stimulation of the subthalamic nucleus. Clin Neurophysiol 115(3):589-595. https://doi.org/10.1016/j.clinph.2003.10.033

Michel E, Hernandez D, Lee SY (2017) Electrical conductivity and permittivity maps of brain tissues derived from water content based on T1-weighted acquisition. Magn Reson Med 77(3):1094-1103

Miller A, Alston R, Corsellis J (1980) Variation with age in the volumes of grey and white matter in the cerebral hemispheres of man: measurements with an image analyser. Neuropathol Appl Neurobiol 6(2):119-132

Moher, D., Liberati, A., Tetzlaff, J., Altman, D. G., \& Group, P (2009) Preferred reporting items for systematic reviews and meta-analyses: the PRISMA statement. PLoS Med 6(7):e1000097

Moher D, Jadad AR, Tugwell P (1996) Assessing the quality of randomized controlled trials: current issues and future directions. Int J Technol Assess Health Care 12(2):195-208

Montes-Restrepo V, van Mierlo P, Strobbe G, Staelens S, Vandenberghe S, Hallez H (2014) Influence of skull modeling approaches on EEG source localization. Brain Topogr 27(1):95-111

Nicholson PW (1965) Specific impedance of cerebral white matter. Exp Neurol 13(4):386. https://doi.org/10.1016/0014-4886(65)90126 $-3$
Oh S, Lee S, Cho M, Kim T, Kim I (2006) Electrical conductivity estimation from diffusion tensor and T2: a silk yarn phantom study. In: Paper presented at the Proc Intl Soc Mag Reson Med

Okada YC, Lahteenmaki A, Xu CB (1999) Experimental analysis of distortion of magnetoencephalography signals by the skull. Clin Neurophysiol 110(2):230-238. https://doi.org/10.1016/s0013 -4694(98)00099-6

Ollikainen JO, Vauhkonen M, Karjalainen PA, Kaipio JP (1999) Effects of local skull inhomogeneities on EEG source estimation. Med Eng Phys 21(3):143-154

Oostendorp TF, Delbeke J, Stegeman DF (2000) The conductivity of the human skull: results of in vivo and in vitro measurements. IEEE Trans on Biomed Eng 47(11):1487-1492. https://doi. org/10.1109/tbme.2000.880100

Opitz A, Windhoff M, Heidemann RM, Turner R, Thielscher A (2011) How the brain tissue shapes the electric field induced by transcranial magnetic stimulation. Neuroimage 58(3):849-859. https:// doi.org/10.1016/j.neuroimage.2011.06.069

Opitz A, Falchier A, Linn GS, Milham MP, Schroeder CE (2017) Limitations of ex vivo measurements for in vivo neuroscience. Proc Natl Acad Sci USA 114(20):5243-5246

Ouypornkochagorn T, Polydorides N, McCann H (2014) In vivo estimation of the scalp and skull conductivity. EIT2015, pp 10

Pal D, Trivedi R, Saksena S, Yadav A, Kumar M, Pandey CM, Rathore RKS, Gupta RK (2011) Quantification of age-and gender-related changes in diffusion tensor imaging indices in deep grey matter of the normal human brain. J Clin Neurosci 18(2):193-196

Pant S, Te T, Tucker A, Sadleir RJ (2011) The conductivity of neonatal piglet skulls. Physiol Meas 32(8): 1275

Park HJ, Kwon JS, Youn T, Pae JS, Kim JJ, Kim MS, Ha KS (2002) Statistical parametric mapping of LORETA using high density EEG and individual MRI: application to mismatch negativities in schizophrenia. Hum Brain Mapp 17(3):168-178. https://doi. org/10.1002/hbm.10059

Pensler J, McCarthy JG (1985) The calvarial donor site: an anatomic study in cadavers. Plast Reconstruct Surg 75(5):648-651

Peterson BS, Anderson AW, Ehrenkranz R, Staib LH, Tageldin M, Colson E, Gore JC, Duncan CC, Makuch R, Ment LR (2003) Regional brain volumes and their later neurodevelopmental correlates in term and preterm infants. Pediatrics 111(5):939-948

Peyman A, Rezazadeh A, Gabriel C (2001) Changes in the dielectric properties of rat tissue as a function of age at microwave frequencies. Phys Med Biol 46(6):1617

Peyman A, Holden S, Watts S, Perrott R, Gabriel C (2007) Dielectric properties of porcine cerebrospinal tissues at microwave frequencies: in vivo, in vitro and systematic variation with age. Phys Med Biol 52(8):2229

Pierpaoli C, Jezzard P, Basser PJ, Barnett A, Di Chiro G (1996) Diffusion tensor MR imaging of the human brain. Radiology 201(3):637-648

Pohlmeier R, Buchner H, Knoll G, Rienacker A, Beckmann R, Pesch $J$ (1997) The influence of skull-conductivity misspecification on inverse source localization in realistically shaped finite element head models. Brain Topogr 9(3):157-162. https://doi. org/10.1007/bf01190384

Rakic P (2006) A century of progress in corticoneurogenesis: from silver impregnation to genetic engineering. Cereb Cortex 16(Suppl_1):i3-i17

Ramon C, Schimpf PH, Haueisen J (2006) Influence of head models on EEG simulations and inverse source localizations. BioMed Eng Online 5(1): 10

Ramon C, Garguilo P, Fridgeirsson EA, Haueisen J (2014) Changes in scalp potentials and spatial smoothing effects of inclusion of dura layer in human head models for EEG simulations. Front Neuroeng. https://doi.org/10.3389/fneng.2014.00032 
Ropella KM, Noll DC (2017) A regularized, model-based approach to phase-based conductivity mapping using MRI. Magn Reson Med 78(5):2011-2021. https://doi.org/10.1002/mrm.26590

Rosenthal R (1991) Quality-weighting of studies in meta-analytic research. Psychother Res 1(1):25-28

Rosenthal RL, Tobias CW (1948) Measurement of the electric resistance of human blood; use in coagulation studies and cell volume determinations. J Lab Clin Med 33(9):1110-1122

Rullmann M, Anwander A, Dannhauer M, Warfield SK, Duffy FH, Wolters CH (2009) EEG source analysis of epileptiform activity using a $1 \mathrm{~mm}$ anisotropic hexahedra finite element head model. Neuroimage 44(2):399-410. https://doi.org/10.1016/j.neuro image.2008.09.009

Rush S, Driscoll DA (1968) Current distribution in the brain from surface electrodes. Anesth Analg 47(6):717-723

Rush S, Driscoll DA (1969) EEG electrode sensitivity-an application of reciprocity. IEEE Trans Biomed Eng 16(1):15-22

Sabancıoğulları V, Koşar Mİ, Şalk İ, Erdil FH, Öztoprak İ, Çimen M (2012) Diploe thickness and cranial dimensions in males and females in mid-Anatolian population: an MRI study. Forensic Sci Int 219(1-3):289

Sadleir RJ, Vannorsdall TD, Schretlen DJ, Gordon B (2010) Transcranial direct current stimulation (tDCS) in a realistic head model. Neuroimage 51(4):1310-1318. https://doi.org/10.1016/j.neuro image.2010.03.052

Salat DH, Kaye JA, Janowsky JS (1999) Prefrontal gray and white matter volumes in healthy aging and Alzheimer disease. Arch Neurol 56(3):338-344

Salinas FS, Lancaster JL, Fox PT (2009) 3D modeling of the total electric field induced by transcranial magnetic stimulation using the boundary element method. Phys Med Biol 54(12):3631-3647. https://doi.org/10.1088/0031-9155/54/12/002

Santos L, Martinho M, Salvador R, Wenger C, Fernandes SR, Ripolles O, Giulio R, Miranda PC (2016) Evaluation of the electric field in the brain during transcranial direct current stimulation: a sensitivity analysis. In: Paper presented at the Engineering in Medicine and Biology Society (EMBC), 2016 IEEE 38th Annual International Conference of the IEEE Engineering in Medicine and Biology Society (EMBC), pp. 1778-1781. IEEE, 2016.

Sastre-Garriga J, Ingle GT, Chard DT, Cercignani M, Ramió-Torrentà L, Miller DH, Thompson AJ (2005) Grey and white matter volume changes in early primary progressive multiple sclerosis: a longitudinal study. Brain 128(6):1454-1460

Schlosser RGM, Nenadic I, Wagner G, Gullmar D, von Consbruch K, Kohler S, Schultz CC, Koch K, Fitzek C, Matthews PM, Sauer $\mathrm{H}$ (2007) White matter abnormalities and brain activation in schizophrenia: a combined DTI and fMRI study. Schizophr Res 89(1-3):1-11. https://doi.org/10.1016/j.schres.2006.09.007

Schmid G, Überbacher R (2005) Age dependence of dielectric properties of bovine brain and ocular tissues in the frequency range of $400 \mathrm{MHz}$ to $18 \mathrm{GHz}$. Phys Med Biol 50(19):4711

Schmidt C, Wagner S, Burger M, van Rienen U, Wolters CH (2015) Impact of uncertain head tissue conductivity in the optimization of transcranial direct current stimulation for an auditory target. $\mathrm{J}$ Neural Eng 12(4):046028

Schmithorst VJ, Wilke M, Dardzinski BJ, Holland SK (2002) Correlation of white matter diffusivity and anisotropy with age during childhood and adolescence: a cross-sectional diffusion-tensor MR imaging study. Radiology 222(1):212-218

Schönborn F, Burkhardt M, Kuster N (1998) Differences in energy absorption between heads of adults and children in the near field of sources. Health Phys 74(2):160-168

Sekino M, Inoue Y, Ueno S (2005) Magnetic resonance imaging of electrical conductivity in the human brain. IEEE Trans Magn 41(10):4203-4205. https://doi.org/10.1109/tmag.2005.854804
Silau AM, Fischer BH, Kjaer I (1995) Normal prenatal development of the human parietal bone and interparietal suture. J Craniofac Genet Develop Biol 15(2):81-86

Skrzat J, Brzegowy P, Walocha J, Wojciechowski W (2004) Age dependent changes of the diploe in the human skull. Folia Morphol 63(1):67-70

Song J, Turovets S, Govyadinov P, Mattson C, Luu P, Smith K, Prior F, Larson-Prior L, Tucker DM (2013) Anatomically accurate infant head models for EEG source localization. Paper presented at the Journal of Physics: Conference Series

Sorensen AG, Wu O, Copen WA, Davis TL, Gonzalez RG, Koroshetz WJ, Reese TG, Rosen BR, Wedeen VJ, Weisskoff RM (1999) Human acute cerebral ischemia: detection of changes in water diffusion anisotropy by using MR imaging. Radiology 212(3):785-792

Stewart-Wallace A (1939) A biochemical study of cerebral tissue, and of the changes in cerebral Ædema. Brain 62(4):426-438

Suh HS, Lee WH, Kim TS (2012) Influence of anisotropic conductivity in the skull and white matter on transcranial direct current stimulation via an anatomically realistic finite element head model. Phys Med Biol 57(21):6961-6980. https://doi. org/10.1088/0031-9155/57/21/6961

Sullivan WG, Smith AA (1989) The split calvarial graft donor site in the elderly: a study in cadavers. Plast Reconstruct Surg 84(1):29-31

Tang C, You FS, Cheng G, Gao DK, Fu F, Yang GS, Dong XZ (2008) Correlation between structure and resistivity variations of the live human skull. IEEE Trans Biomed Eng 55(9):2286-2292. https://doi.org/10.1109/tbme.2008.923919

Tha KK, Katscher U, Yamaguchi S, Stehning C, Terasaka S, Fujima N, Kudo K, Kazumata K, Yamamoto T, Van Cauteren M (2018) Noninvasive electrical conductivity measurement by MRI: a test of its validity and the electrical conductivity characteristics of glioma. Eur Radiol 28(1):348-355

Todd TW (1924) Thickness of the male white cranium. Anat Rec 27(5):245-256

Tuch DS, Wedeen vJ, Dale AM, George JS, Belliveau JW (1999) Conductivity mapping of biological tissue using diffusion MRI. Ann N Y Acad Sci 888(1):314-316

Tuch DS, Wedeen VJ, Dale AM, George JS, Belliveau JW (2001a) Conductivity tensor mapping of the human brain using diffusion tensor MRI. Proc Natl Acad Sci USA 98(20):11697-11701

Tuch DS, Wedeen VJ, Dale AM, George JS, Belliveau JW (2001b) Conductivity tensor mapping of the human brain using diffusion tensor MRI. Proc Natl Acad Sci 98:11697-11701

Uluğ AM, Van Zijl PC (1999) Orientation-independent diffusion imaging without tensor diagonalization: anisotropy definitions based on physical attributes of the diffusion ellipsoid. J Magn Reson Imag 9(6):804-813

van den Broek SP, Reinders F, Donderwinkel M, Peters M (1998) Volume conduction effects in EEG and MEG. Electroencephalogr Clin Neurophysiol 106(6):522-534

Van Lier A, Hoogduin J, Polders D, Boer V, Hendrikse J, Robe P, Woerdeman PA, Lagendijk JJ, Luijten PR, van den Berg C (2011) Electrical conductivity imaging of brain tumours. Paper presented at the Proceedings of the 19th Annual Meeting of ISMRM, Montreal, Canada

van Lier A, Kolk A, Brundel M, Hendriske J, Luijten J, Lagendijk J, van den Berg C (2012) Electrical conductivity in ischemic stroke at 7.0 Tesla: a case study. Paper presented at the Proceedings of the 20th Scientific Meeting of the International Society of Magnetic Resonance in Medicine (ISMRM'12)

Vatta F, Bruno P, Inchingolo P (2002) Improving lesion conductivity estimate by means of EEG source localization sensitivity to model parameter. J Clin Neurophysiol 19(1):1-15. https://doi. org/10.1097/00004691-200201000-00001 
Verhagen AP, de Vet HC, de Bie RA, Boers M, van den Brandt PA (2001) The art of quality assessment of RCTs included in systematic reviews. J Clin Epidemiol 54(7):651-654

Vijay Kumar A, Agarwal S, Bastia B, Shivaramu M, Honnungar R (2012) Fusion of skull vault sutures in relation to age-a cross sectional postmortem study done in $3 \mathrm{rd}$, 4th \& 5th decades of life. J Forensic Res 3(10):2145-2157

Voigt T, Doessel O, Katscher U (2009) Imaging conductivity and local SAR of the human brain. In: Paper presented at the Proceedings of the 17th Annual Meeting of ISMRM, Honolulu, USA

Voigt T, Katscher U, Doessel O (2011) Quantitative conductivity and permittivity imaging of the human brain using electric properties tomography. Magn Reson Med 66(2):456-466. https://doi. org/10.1002/mrm.22832

Vorwerk J, Cho JH, Rampp S, Hamer H, Knosche TR, Wolters CH (2014) A guideline for head volume conductor modeling in EEG and MEG. Neuroimage 100:590-607. https://doi.org/10.1016/j. neuroimage. 2014.06.040

Vu HL, Panchal J, Parker EE, Levine NS, Francel P (2001) The timing of physiologic closure of the metopic suture: a review of 159 patients using reconstructed 3D CT scans of the craniofacial region. J Craniofac Surg 12(6):527-532

Wang K, Zhu S, Mueller BA, Lim KO, Liu Z, He B (2008) A new method to derive white matter conductivity from diffusion tensor MRI. IEEE Trans Biomed Eng 55(10):2481-2486

Wang Q, Xu X, Zhang M (2010) Normal aging in the basal ganglia evaluated by eigenvalues of diffusion tensor imaging. Am J Neuroradiol 31(3):516-520

Wendel K, Malmivuo J, Ieee (2006) Correlation between live and post mortem skull conductivity measurements

Wenger C, Salvador R, Basser PJ, Miranda PC (2015) The electric field distribution in the brain during TTFields therapy and its dependence on tissue dielectric properties and anatomy: a computational study. Phys Med Biol 60(18):7339

Whiting P, Rutjes AW, Reitsma JB, Bossuyt PM, Kleijnen J (2003) The development of QUADAS: a tool for the quality assessment of studies of diagnostic accuracy included in systematic reviews. BMC Med Res Methodol 3(1):25

Wise T, Radua J, Via E, Cardoner N, Abe O, Adams T, Amico F, Cheng Y, Cole JH, de Perico CAM (2017) Common and distinct patterns of grey-matter volume alteration in major depression and bipolar disorder: evidence from voxel-based meta-analysis. Mol Psychiatry 22(10): 1455

Wolters CH, Anwander A, Tricoche X, Weinstein D, Koch MA, MacLeod RS (2006) Influence of tissue conductivity anisotropy on EEG/MEG field and return current computation in a realistic head model: a simulation and visualization study using highresolution finite element modeling. Neuroimage 30(3):813-826. https://doi.org/10.1016/j.neuroimage.2005.10.014

Wu Y, Koch W, Pratt K (1991) Proposed new electrolytic conductivity primary standards for $\mathrm{KCl}$ solutions. J Res Natl Inst Stand Technol 96(2):191

Wu Z, Liu Y, Hong M, Yu X (2018) A review of anisotropic conductivity models of brain white matter based on diffusion tensor imaging. Med Biol Eng Comput 56(8):1325-1332

Youn T, Park H-J, Kim J-J, Kim MS, Kwon JS (2003) Altered hemispheric asymmetry and positive symptoms in schizophrenia: equivalent current dipole of auditory mismatch negativity. Schizophr Res 59(2):253-260

Zhang YC, van Drongelen W, He B (2006) Estimation of in vivo brain-to-skull conductivity ratio in humans. Appl Phys Lett 89(22):223903. https://doi.org/10.1063/1.239888

Zhang X, de Moortele PFV, Schmitter S, He B (2013) Complex B1 mapping and electrical properties imaging of the human brain using a 16-channel transceiver coil at 7T. Magn Reson Med 69(5):1285-1296

Publisher's Note Springer Nature remains neutral with regard to jurisdictional claims in published maps and institutional affiliations. 